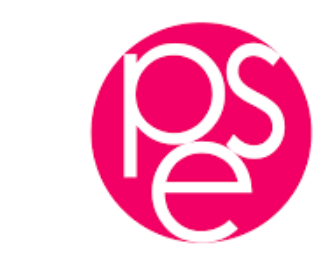

PARISSCHOOL OFECONOMICS
ECOLED'ECONOMIEDE PARIS

WORKING PAPER Nº 2020 - 31

Diffusion of soil pollution in an agricultural economy. The emergence of regions, frontiers and spatial patterns.

Carmen Camacho

Alexandre Cornet

JEL Codes: C61, 044, Q15, Q56, R11.

Keywords: Spatial growth, Diffusion, Soil Pollution, Frontier, Dynamic programming, Optimal Control.

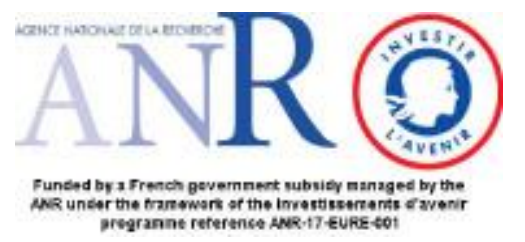




\title{
Diffusion of soil pollution in an agricultural economy. \\ The emergence of regions, frontiers and spatial patterns.
}

\author{
Carmen Camacho \\ Alexandre Cornet \\ PJSE UMR8545, PSE \\ Université Paris 1
}

March 28, 2021

\begin{abstract}
The Status of the World's Soil Resources Report identified in 2015 soil pollution as a major threat to all the services provided by soils (FAO and ITPS, 2015). This paper develops a spatial growth model for an agricultural economy where pollution diffuses in the soil. In order to produce, the economy needs fertile soil, which is naturally bounded by the amount of available land. Although production entails pollution, locations can protect their soil investing in abatement. Once a location reaches its maximum of fertile land, the economy is split into a fertile region and a polluted region, separated by a dynamic frontier which follows the spatial evolution of pollution. After providing the optimal trajectories for consumption and fertile land, we prove that heterogenous steady states can emerge even in homogeneous economies, and that a polluted region can stagnate forever in an environmental poverty trap. Our results are numerically illustrated, including examples of the economy's resilience to pollution shocks.
\end{abstract}

Keywords: Spatial growth, Diffusion, Soil Pollution, Frontier, Dynamic programming, Optimal Control.

Journal of Economic Literature: C61, O44, Q15, Q56, R11. 


\section{Introduction}

Every year, agriculture is responsible for a loss of 24 billion tonnes of fertile soil (UNCCD, 2017). This loss implies major risks in food security and crop sustainability worldwide. There is an urgent need to understand and foresee the impact of agriculture on soil fertility since food demand will keep increasing in the next decades, pushed by population growth. Although research on the economics of soil loss started in the late 1960's, this area has attracted relatively little attention until a very recent past. As Burt (1981) pointed out, "the most obvious reason for this apparent lack of interest in the subject is the view that advances in technology have made soil resources per se of less consequence for agricultural production". Indeed, in most economic growth papers, agricultural production is simply modeled as a linear function of land, implying that increases in technology or land lead to proportional increases in production. However, we know that regions can actually remain below their production capacity, despite technology and the possibility to increase soil fertility. In this paper, we develop a spatial growth model to account for the diffusion of pollution in agricultural soils. With the analytical optimal trajectories for fertile and polluted land in hand, we can measure how local pollution affects all other locations' fertility.

Soil pollution can be defined as "the presence of a chemical or substance out of place and/or present at a higher than normal concentration that has adverse effects on any nontargeted organism" (ITPS, 2015). ${ }^{1}$ Generally speaking, anthropogenic activities are the main source of soil pollution and unfortunately, there is no lack of examples: wars, mining, former factory sites, accidental oil leakage, over use of fertilizers, etc. Concerns are now growing since the Status of the World's Soil Resources Report identified soil pollution as one of the main threats to all the services provided by soils ecosystems (FAO and ITPS, 2015). Soil pollution falls in two categories: point-source and diffuse. On the one hand, point source pollution is "released to the soil, and the source and identity of the pollution is easily identified" (FAO, 2018). To illustrate point soil pollution, let us mention inadequate waste disposal or accidental chemical leakages. On the other hand, diffuse pollution is "spread over very wide areas, accumulates in soil, and does not have a single or easily identified source". Regarding the agricultural activity, the use of fertilizers is the main source of diffuse pollution. This paper focuses on soil pollution caused by inappropriate agricultural practices as the over use of fertilizers and, as a result, it will only consider diffuse soil pollution.

Diffuse soil pollution is mainly originated from inappropriate agricultural practices, and in particular from the use of nitrogenous fertilizers. Although we now have a precise understanding on how nitrogenous fertilizers pollute soils and even drinkable water, their use in

\footnotetext{
1 "Soil pollution" shall not be used as a synonym for "soil contamination". For the ITPS a soil is contaminated when "the concentration of a chemical or substance is higher than would occur naturally but is not necessarily causing harm" (ITPS, 2015).
} 
agriculture keeps increasing every year. In 1980, the worldwide nitrogenous fertilizer consumption attained 60 million tonnes, and almost doubled in less than 40 years, reaching 110 millions tonnes in 2014. It goes without saying that the use of fertilizers depends on local regulations. For instance, due to a lack of a strict environmental regulation in China, 19\% of all of its agricultural soil is officially declared as polluted by the Chinese Environmental Protection Ministry (CCICED, 2015). In contrast, after an even more important growth in the use of fertilizers in Europe between 1960 and 1980, their use abruptly decreased by almost $50 \%$ by 1990, and it has been decreasing ever since. The decrease in the use of nitrogenous fertilizers in Europe can directly be related to the EU Nitrate Directive of 1991 which aimed at "preventing nitrates from agricultural sources polluting ground and surface waters and by promoting the use of good farming practices" (EU Commission, 1991). There is no doubt that legislations can make a real difference regarding the pollution in soils, and this is why we adopt here the perspective of a policy maker.

The economics of soil conservation has a long tradition of interdisciplinary thinking, borrowing tools and concepts from related disciplines as ecology or agronomy. In the early days of soil economics, Ciriacy-Wantrup (1968) borrowed from ecology the concept of damage thresholds to study irreversible damages due to agricultural production. Nevertheless, as already mentioned, soil conservation did not receive the required attention for a long time. A second wave of theoretical research on the economics of soil developed from the 1980's to analyze the complex interactions between agricultural practices and soil fertility. The seminal paper of Pope et al. (1983) analyzes the relationships among soil loss, topsoil depth, net farm income, and technological progress and provide with the optimal policies in soil conservation which maximizes net farm income. Similarly, Saliba (1985) studies the interactions between the choices in soil management, soil loss and farmland productivity. Noteworthy, among the papers of this second wave, there are region specific case-studies like Segarra et al. (1987) and Barbier (1990). More recently in the 2000s, the question of soil conservation shifted to the context of developing countries where it is thought that better soil management practices could lead to the highest potential gains. ${ }^{2}$

As pointed out, the economics of soil conservation touches upon different trends in economics as agricultural, environmental, development economics and dynamic programming. Here we study the economics of soil conservation using a spatial growth model where the diffusion of pollution is described using Fick's law. According to Fick's law the flux of pollution concentration at a location is proportional to the concentration gradient of pollution at this location (see Brock and Xepapadeas, 2008 and 2010, Smith et al., 2009, La Torre et al, 2015, or Camacho and Perez-Barahona, 2015). Our agricultural economy is made of a

\footnotetext{
${ }^{2}$ See for instance Antle et al. (2006), Hagos et al. (2006), de Graaf et al. (2008), Stephens et al. (2012), Barrett et al. (2015), Bevis et al. (2017), or Berazneva et al. (2018).
} 
continuum of locations distributed along the unit circle, and each location is endowed with a given amount of fertile soil. The unique agricultural good is locally managed, and it can be either directly consumed or reinvested to abate at least some of the pollution generated. Our model is structurally close to Boucekkine et al. (2013) and Boucekkine et al. (2018), since production is linear in fertile soil. Besides, pollution locally generated can diffuse across locations as in Camacho and Perez-Barahona (2015). And yet, there is a key difference with all existing spatial models which study the diffusion of wealth or air pollution: the production factor, fertile soil, is naturally bounded.

The explicit optimal solution foresees the economy transiting through two distinct phases. In the first phase all locations have some pollution and the policy maker takes into account locations' heterogeneity and optimal consumption at a given location will depend, among other factors, on its abatement efficiency. Despite being spatially heterogeneous, consumption grows at the same constant rate at all locations as in Boucekkine et al. (2018). A second phase is triggered when all land is fertile in at least one location. In this second phase, the economy is divided in one fertile region and a polluted region. Hence, the two regions are separated by a pollution frontier which evolves over time and space, responding to the optimal decisions taken in both the polluted and fertile region.

Locations in the fertile region abate their own pollution, but only theirs, putting an end to global diffusion and their role of pollution sinks. Outside the realm of fertility, some locations optimally continue emitting beyond their abatement capacity and pollution diffuses. Contrary to the well-known results that apply when the production factors are unbounded, the economy can reach a steady state with two distinct regions: a permanently fertile region, and next to it, a polluted region. How can such a situation arise? During the first phase, the more fertile locations act as pollution sinks, absorbing pollution generated at the less fertile locations. Once fertile locations attain the second phase and stop acting as pollution sinks, polluted locations may not be able to abate enough to eradicate their own pollution. If this happens, the polluted region stagnates forever at low levels of fertile soil, never catching up with the fertile region.

From a modeling perspective, this paper describes for the first time the evolution of an economy made of two regions separated by a frontier that can evolve geographically with time, responding to the policy maker's optimal decisions. It also provides with numerical exercices and show that in a spatially homogeneous economy, initial disparities in soil fertility may not vanish with time if the fertile region does not help absorbing the pollution from neighboring locations. Moreover, we also assess numerically the resiliency of the economy to pollution shocks. Overall, we show that if the economy uses a sufficiently advanced technology, then the pollution shock can be absorbed with time via abatement. In this case, the economy progressively turns back to its initial fully fertile state. However, if technology is 
not sophisticated enough, then the pollution shock can diffuse across the economy, at the risk of affecting a too large area of the economy, leading to a global collapse.

The rest of the paper is organized as follows. The model is presented in section 2. Section 3 provides with the optimal solution for the policy maker problem, describing the transit between the two phases of the optimal solution. In Section 4 we run different numerical exercises, which show some dynamic properties of the model. Section 5 concludes. All the proofs are gathered in the Appendix.

\section{Soil pollution diffusion in a linear growth model}

We consider a closed economy, where both land and households are distributed over the unit circle on the plane, $\mathcal{S}=\left\{(\sin \theta, \cos \theta) \in \mathbb{R}^{2}: \theta \in[0,2 \pi]\right\}$. Each location $\theta \in \mathcal{S}$ is populated by $N(\theta)$ individuals and is endowed with an amount of land $L(t, \theta)$.

Assumption 1. The spatial distribution of land, $L(\cdot)$ is given and independent of time. Moreover, $L(\cdot)$ is either a constant or a linear function of space.

Assumption 1 implies that locations cannot increase their total land allocation. We also assume that land is composed of both fertile and polluted soil, $L_{F}$ and $L_{P}$, that is, $L(\theta)=L_{P}(t, \theta)+L_{F}(t, \theta)$. All locations produce a unique agriculture good from the labour of local fertile soil, according to the following linear production function $Y(t, \theta)=A(\theta) L_{F}(t, \theta)$, where $A(\theta)$ is the local production technology at location $\theta$. Hence, a partially polluted location can still produce.

The dynamics of soil pollution at one location is explained by three factors. First, pollution diffuses accordingly to Fick's law: pollution diffuses from more polluted locations to less polluted locations and its flux is proportional to the pollution gradient. Accordingly, the diffusion of soil pollution is captured by $D \frac{\partial^{2} L_{P}}{\partial \theta^{2}}(t, \theta)$, where $D$ is the diffusion coefficient. For simplicity reasons, $D$ is assumed both constant in time and homogeneous in space. ${ }^{3}$ Second, fertile soil deteriorates locally. Indeed, local production generates some pollutant, which transforms fertile soil into polluted soil. The local effect is measured as $\nu(\theta) A(\theta) L_{F}(t, \theta)$, where $\nu(\theta)$ is the local sensitivity of fertile soil to pollution. $\nu(\theta)$ can be related to more or less pollutant production technologies, to different levels of biodiversity, etc. And third, soil pollution is reversible. Indeed, polluted soil can be depolluted independently of the local pollution level. This last assumption is made for simplicity, being aware that it would be more accurate to consider that abatement is feasible only if pollution remains below a certain

\footnotetext{
${ }^{3}$ We do not consider any seasonal effect nor heterogeneity in soil porosity, which would lead to study time and space dependent diffusion coefficients. These more general specifications for the diffusion coefficient could be analyzed following Boucekkine et al. (2020), but it is out of the scope of this paper.
} 
threshold. ${ }^{4}$ Letting $C(t, \theta)$ denote total consumption at location $\theta$ at time $t$, the amount invested in abatement at location $\theta$ is $A(\theta) L_{F}(t, \theta)-C(t, \theta) \geq 0$. Let $\phi(\theta)$ be the local pollution abatement efficiency. Then putting together the three factors behind local pollution, the spatial dynamics of polluted soil can be described as

$$
\frac{\partial L_{P}}{\partial t}(t, \theta)=D \frac{\partial^{2} L_{P}}{\partial \theta^{2}}(t, \theta)+\nu(\theta) A(\theta) L_{F}(t, \theta)-\phi(\theta)\left[A(\theta) L_{F}(t, \theta)-C(t, \theta)\right] .
$$

Under Assumption 1, one can write that $\frac{\partial L_{F}}{\partial t}(t, \theta)=-\frac{\partial L_{P}}{\partial t}(t, \theta)$, and writing total consumption $C(t, \theta)$ as the product of per capita consumption, $c(t, \theta)$, and the location's time-independent population $N(\theta)$, the evolution of fertile soil becomes

$$
\left\{\begin{array}{l}
\frac{\partial L_{F}}{\partial t}(t, \theta)=D \frac{\partial^{2} L_{F}}{\partial \theta^{2}}(t, \theta)+A(\theta)[\phi(\theta)-\nu(\theta)] L_{F}(t, \theta)-c(t, \theta) N(\theta) \phi(\theta), \\
L_{F}(0, \theta)=L_{F}^{0}(\theta),
\end{array}\right.
$$

for all $t>0$ and $\theta \in \mathcal{S}$, where $A(\theta)[\phi(\theta)-\nu(\theta)]$ is the net land productivity. From now on we will only work with $L_{F}$. Obviously, it is straightforward to obtain the corresponding results for $L_{P}$.

Next, let us introduce the linear operator $\mathcal{L}$ defined for all functions $u$ in the Hilbert space of function from $\mathcal{S}$ to $\mathbb{R}$ by, $\mathcal{L} u(\theta):=D u^{\prime \prime}(\theta)+A(\theta)[\phi(\theta)-\nu(\theta)] u(\theta)$. Using $\mathcal{L},(1)$ can be rewritten as

$$
\frac{\partial L_{F}}{\partial t}(t, \theta)=\mathcal{L} L_{F}(t, \theta)-c(t, \theta) N(\theta) \phi(\theta)
$$

where the initial distribution of $L_{F}$ is known, $L_{F}(0, \theta)=L_{F}^{0}(\theta)$ for all $\theta \in \mathcal{S}$. Operator $\mathcal{L}$ is well behaved when applied to twice differentiable functions, measurable in $\mathcal{S}$. We say a function $\phi$ defined on $\mathcal{S}$, regular and non-identically zero, is an eigenfunction of $\mathcal{L}$, with associated eigenvalue $\lambda \in \mathbb{R}$ if $\mathcal{L} \phi(x)=\lambda \phi(x)$. Coddington and Levinson (1955) prove that there exists a countable set of eigenvalues $\left\{\lambda_{n}\right\}_{n \geq 0}$, which can be ordered as a decreasing sequence. It can be proven that the first eigenvalue of $\mathcal{L}, \lambda_{0}$, is an eigenvalue with multiplicity 1 , all other eigenvalues have either multiplicity 1 or $2 .{ }^{5}$ Besides, $e_{0}$, the eigenfunction associated to $\lambda_{0}$, is strictly positive on the unit circle and one can set $\int_{0}^{2 \pi} e_{0}^{2}(\theta) d \theta=1$. Moreover, the eigenfunctions of $\mathcal{L}$ form an orthonormal basis in $L^{2}(\mathcal{S})$ and the sequence of eigenvalues tends to $-\infty$, while the eigenfunction $e_{n}$ associated to $\lambda_{n}$ has $n$ zeros in $[0,2 \pi]{ }^{6}$

Finally, we assume there exists a policy maker, whose aim is to maximize overall welfare. Welfare is measured as the present value of the spatial aggregate of individuals' utility. Here,

\footnotetext{
${ }^{4}$ The critical zone for pollution reversibility has been widely studied, see for instance Dupouey et al. (2002), Chartier et al. (2006), Gao et al. (2011) and Le Kama et al. (2014). Technically speaking, introducing irreversible pollution damages would lead us to impose that above a local pollution threshold concentration, the first partial derivative of fertile soils with respect to time should be negative, that is $\frac{\partial L_{F}(t, \theta)}{\partial t} \leq 0$.

${ }^{5}$ The multiplicity of an eigenvalue is the number of times it appears in the sequence $\left\{\lambda_{n}\right\}_{n \geq 0}$.

${ }^{6}$ See Appendix A for a brief introduction to abstract dynamic systems in infinite dimensions. For further details see Coddinton and Levinson (1955) or Brown et al. (2013).
} 
utility depends solely on consumption per capita, $c$, and it is measured by a constant intertemporal elasticity of substitution function of parameter $\sigma \in \mathbb{R}$. Knowing that the policy maker discounts time at a constant rate $\rho$, her problem writes as

$$
\max _{c} \int_{0}^{\infty} e^{-\rho t}\left[\int_{0}^{2 \pi} \frac{c(t, \theta)^{1-\sigma}}{1-\sigma} N(\theta) d \theta\right] d t
$$

subject to (1) and

$$
\begin{aligned}
0 & \leq L_{F}(t, \theta) \leq L(\theta), \\
c(t, \theta) & \geq 0, \text { for all } \theta \in \mathcal{S}, \text { and } t \geq 0 .
\end{aligned}
$$

Our model is structurally close to Boucekkine et al. (2013) and Boucekkine et al. (2018), since production is linear in fertile soil. As in previous works, all land is labored at all locations, independently of the distribution of population. Nevertheless, there is a key difference with all previous work in the literature: the production factor, here fertile soil, is naturally bounded. We define the different phases an economy may go through:

Definition 1. The economy is said to be in Phase 1 if the constraint for fertile soil in (4) is not binding. Similarly, the economy is in Phase 2 if (4) binds.

We face here new challenges. First, our problem requires the description of optimal trajectories in two separate phases. It is worth noting that the policy maker will play an active role in Phase 1 by indirectly redistributing consumption from fertile to polluted locations. And second, we need to provide a description of the dynamic frontier dividing the fertile region and the partially polluted region in Phase 2. In this regard, we will invoke Stefan's equation to describe the evolution of our frontier as a function of the gradient of pollution. A detailed description is provided in Section 3.2.

\section{Optimal policy and the evolution of fertile soil}

This section provides with the optimal solution to the policy maker problem presented in Section 2. In particular, we show that the economy optimally transits through two phases. In Phase 1, each location's land is divided between fertile and polluted soil. We will prove that during Phase 1, fertile soil steadily grows at a constant rate. Phase 2 is triggered whenever fertile soil at a single location attains its maximum or its minimum. The following subsections characterize the entire paths for optimal consumption and fertile soil. ${ }^{7}$

\footnotetext{
${ }^{7}$ We have relegated to the Appendix most technical material as well as all the article's proofs.
} 


\subsection{Optimal dynamics in the polluted economy}

As in Boucekkine et al. (2018), the following assumption ensures both the positivity of the value function and the finiteness of the inter-temporal utility:

Assumption 2. $\rho>\lambda_{0}(1-\sigma)$, where $\lambda_{0}$ the first eigenvalue of the problem $\mathcal{L} u=\lambda u$.

Let $\left\langle L_{F}\right\rangle(t):=\frac{1}{2 \pi} \int_{0}^{2 \pi} L_{F}(\eta, t) d \eta$ be the instantaneous spatial mean value for a given fertile soil distribution $L_{F}$ at time $t$. We provide next the optimal solution for fertile soil and consumption in Phase 1.8

Proposition 1. Under Assumptions 1 and 2, the optimal spatial dynamics of fertile soil in Phase $1, L_{F}^{*}(t, \theta)$, is the solution to

$$
\frac{d L_{F}^{*}}{d t}(t, \theta)=\mathcal{L} L_{F}^{*}(t, \theta)-[\phi(\theta) N(\theta)]^{\frac{\sigma-1}{\sigma}} N(\theta)^{\frac{1}{\sigma}}\left(\alpha_{0} e_{0}(\theta)\right)^{-\frac{1}{\sigma}} \int_{0}^{2 \pi} L_{F}^{*}(t, \eta) \alpha_{0} e_{0}(\theta) d \eta
$$

where $L_{F}^{*}(t, \theta)<L(\theta)$ for all $t \geq 0$ and $\theta \in \mathcal{S}$. At any time $t$, the mean of optimal fertile land is

$$
\left\langle L_{F}^{*}\right\rangle(t)=\left\langle L_{F}^{0}\right\rangle e^{g t}
$$

and $L_{F}^{0}(\theta)=L_{F}(0, \theta)$ is the initial distribution of fertile soil. Moreover, optimal consumption $c^{*}$ can be expressed as

$$
c^{*}(t, \theta)=\left[\phi(\theta) \alpha_{0} e_{0}(\theta)\right]^{-\frac{1}{\sigma}} e^{g t} \int_{0}^{2 \pi} L_{F}^{0}(\eta) \alpha_{0} e_{0}(\theta) d \eta,
$$

where $\alpha_{0} \in \mathbb{R}$ is defined as

$$
\alpha_{0}=\left[\frac{\sigma}{\rho-\lambda_{0}(1-\sigma)} \int_{0}^{2 \pi}\left[\phi(\eta) e_{0}(\eta)\right]^{-\frac{1-\sigma}{\sigma}} N(\eta) d \eta\right]^{\frac{\sigma}{1-\sigma}} .
$$

$e_{0}$ is the first eigenvector of the problem $\mathcal{L} u=\lambda u$ associated to the first eigenvalues $\lambda_{0}$, and $g=\frac{\lambda_{0}-\rho}{\sigma}$ is the growth rate of the economy.

Proposition 1 proves that both the mean of fertile soil and consumption grow at a constant and homogeneous rate $g$ from $t=0$. Hence, although consumption varies across locations, it grows at the same constant rate everywhere. If $g$ is positive, then consumption increases continuously with time. However, note that fertile soil cannot increase forever because land is naturally bounded. Some locations will attain their maximum for fertile land and trigger

\footnotetext{
${ }^{8}$ Note that although we have separated the presentation of the optimal solution in the two phases for the sake of exposition, the optimization problem is solved globally from $t=0$ taking into account the eventuality of reaching the upper bound for $L_{F}$ at certain locations (see Appendix B). Using the optimal conditions found in Appendix B, Appendix C finds the explicit optimal solution in Phase 1, while Appendix F provides us with the optimal solution in Phase 2 that we provide in Section 3.2.
} 
Phase 2, which we study in next section. If $g<0$, then fertile soil tends to zero at all locations, and according to Definition 1, the economy could also enter Phase 2. Although we will focus on the case of a second phase with $g>0$, this other case could be studied along the same lines.

Proposition 1 also reveals the existence of a sustainable level for the time discount rate $\tilde{\rho}=\lambda_{0}$. If the policy maker weights the future generations enough, namely if $\rho<\tilde{\rho}$, then she sacrifices current consumption to increase abatement and guarantee future generations' consumption. In this case $g>0$, and at least some locations attain the maximum of fertile soil. If $\rho=\tilde{\rho}$, then $g=0$ and the economy remains forever at the initial situation. When the policy maker is not altruistic enough and $\rho>\tilde{\rho}$, then $g<0$. Here, the policy maker privileges consumption, reduces abatement, seriously endangering the future of fertile soil, production and consumption.

As in Boucekkine et al. (2018), the policy maker cannot disregard the heterogeneity of the economy. Despite weighting equally the welfare of each individual, consumption per capita varies across locations. Note that $c^{*}$ in (6) depends on the location characteristics via $\phi$ and $e_{0}$. However, since the analytical form of $e_{0}$ is not usually computable, we cannot provide a detailed analytical description of the effect of the different factors on $c^{*}$. Similarly, when $A, \phi, \nu$ and $N$ are spatially distributed and $D \neq 0$, the growth rate $g$ depends on the first eigenvalue $\lambda_{0}$ of $\mathcal{L}$, which generally does not have a closed form.

Fortunately, we can provide closed form expressions for the eigenvectors and eigenvalues of the operator under specific hypothesis on the model's parameters. As a result, closed forms will also obtain for the growth rate, consumption and fertile soil. In particular, we study next two applications of the general framework for which solutions are analytically tractable. In the first one, $A, \phi, \nu$ and $N$ are homogeneous and where the initial distribution of fertile soil is potentially heterogeneous. In the second application, we analyse the role of diffusion by comparing the previous results to an unconnected economy where pollution does not diffuse across locations, i.e. where $D=0$.

Proposition 2. Let Assumptions 1 and 2 hold and let us consider a diffusive economy where $A, \phi, \nu$ and $N$ are homogeneously distributed in space. The optimal trajectory for fertile soil in Phase 1 is

$$
L_{F}^{*}(t, \theta)=\left\langle L_{F}^{0}\right\rangle e^{g t}+\sum_{n \geq 1}\left\langle L_{F}^{0}, e_{n}\right\rangle e_{n}(\theta) e^{\lambda_{n} t}, \quad \forall \theta \in \mathcal{S} \text { and } \forall t \in[0, \tau[
$$

where $L_{F}^{0}$ is the initial distribution of fertile soil, $\lambda_{n}$ the $n^{\text {th }}$ eigenvalue of the problem $\mathcal{L} u=\lambda u$ with associated eigenvector $e_{n}$, with $\lambda_{n}=A(\phi-\nu)-D n^{2}$ for $n \geq 0$, and $\tau$ the time at which the economy reaches Phase 2.

Moreover, the optimal growth rate of consumption is independent of the pollution diffusion 
coefficient $D$ and of population $N$

$$
g=\frac{\lambda_{0}-\rho}{\sigma}=\frac{A(\phi-\nu)-\rho}{\sigma} .
$$

Proposition 2 provides a very convenient writing for the optimal distribution of fertile soil. Indeed, $L_{F}^{*}$ is the sum of its mean value, $\left\langle L_{F}^{0}\right\rangle e^{g t}$, and a second term, $\sum_{n \geq 1}\left\langle L_{F}^{0}, e_{n}\right\rangle e_{n} e^{\lambda_{n} t}$, with zero mean. The effects of pollution are all contained in the second term, and in particular, in the eigenvalues $\lambda_{n}$. Note that if diffusion is strong enough, and here strong enough means that $D>A(\phi-\nu)$, then $\lambda_{n}<0$ for $n \geq 1$ and the second term vanishes with time. Hence if soil is permeable enough, then initial disparities disappear with time and all locations join the same trajectory.

Next let us consider a homogeneous unconnected economy where $D=0$. Here, soil pollution no longer diffuses so that the activity of one location does not affect its neighbors. Hence the optimal control problem presented in Section 2 reduces to a much simpler standard problem, which can be solved for each location independently. In what follows we analyze and quantify the gains and losses of belonging to a diffusive economy with positive growth. The consumption transfer distribution can be defined as

$$
\Gamma(t, \theta):=c_{U}(t, \theta)-c_{D}(t, \theta)
$$

where $c_{U}$ and $c_{D}$ stand for consumption in the unconnected and in the diffusive economy, respectively. In other words, $\Gamma$ measures the losses in consumption from entering a diffusive economy. Whenever $\Gamma(t, \theta)$ is positive, it means that location $\theta$ would be better off alone at time $t$. Obviously, since the contrary is also true, the economy will be made of losers and winners at all times. Will there be an actual and direct transfer of consumption? The answer is no. Recall that the agricultural good cannot be traded nor shipped among locations. Then, what do we mean by consumption transfer? An indirect transfer in consumption is generated when a location makes an extra effort in abatement, acting as a pollution sink and allowing the neighboring locations to increase their own consumption. The following proposition measures the exact amount of this indirect transfer:

Proposition 3. When $A, \phi, \nu$ and $N$ are homogeneous, the consumption profiles are

$$
\begin{aligned}
& c_{U}(t, \theta)=\frac{\rho+A(\phi-\nu)(\sigma-1)}{N \sigma \phi} L_{F}^{0}(\theta) e^{g t}, \\
& c_{D}(t, \theta)=\frac{\rho+A(\phi-\nu)(\sigma-1)}{N \sigma \phi}\left\langle L_{F}^{0}\right\rangle e^{g t} .
\end{aligned}
$$

$L_{F}^{0}$ is the initial distribution of fertile soil and $\left\langle L_{F}^{0}\right\rangle$ its mean value. The two consumption profiles grow at the same rate $g=\frac{A(\phi-\nu)-\rho}{\sigma}$, and the consumption transfer distribution depends 
on the initial distribution of fertile soil $L_{F}^{0}(\theta)$

$$
\Gamma(t, \theta)=\frac{\rho+A(\phi-\nu)(\sigma-1)}{N \sigma \phi}\left[L_{F}^{0}(\theta)-\left\langle L_{F}^{0}\right\rangle\right] e^{g t} .
$$

Proposition 3 proves that whether pollution diffuses or not, homogeneous economies grow at the same rate. In both cases, if the net productivity $A(\phi-\nu)$ is not large enough, that is if $A(\phi-\nu)<\rho$, then $g<0$ and consumption decreases. It is worth to interpret this inequality in terms of $\phi$. It turns out that there exists an abatement efficiency threshold, $\frac{\rho}{A}+\nu$ above which the economy prospers, and below it collapses. From here, we can obtain an insightful conclusion: an economy which wants to grow and enjoy ever increasing consumption needs first to adopt a performing abatement technology and devote enough resources to preserve land.

Although the growth rates of consumption are identical in both economies, their distributions differ. In the unconnected economy, consumption depends solely on the local initial endowment of fertile soil and spatial inequalities in consumption persist in time. Note how consumption grows at a constant rate $g$ without ever adjusting to current values of fertile soil, only building on the initial land endowment. In contrast, consumption in the diffusive economy is homogeneous in space, even starting from an heterogeneous initial distribution $L_{F}^{0}$. Finally, Assumption 2 implies that $\rho+A(\phi-\nu)(\sigma-1)>0$. Therefore, if $L_{F}^{0}(\theta)$ is above the economy's initial mean value, then $\Gamma(t, \theta)>0$ and location $\theta$ will always transfer consumption. Hence Proposition 3 reveals that the policy maker induces a larger abatement effort to the initially more fertile and productive locations so that they can absorb pollution from other locations, which thanks to that, can increase their consumption.

\subsection{The fertile, the polluted region and their dynamic frontier}

The second phase begins the moment one location's fertile soil attains its maximum. Locations at the maximum divide their production between consumption and the abatement of instantaneously generated pollution to ensure that fertile soil remains at its maximum.

Let $\mathcal{S}=\underline{\mathcal{S}}(t) \cup \overline{\mathcal{S}}(t)$, where $\overline{\mathcal{S}}(t)$ is made of the locations that have reached the upper bound for fertility at time $t$, and $\underline{\mathcal{S}}(t)$ of those which have not reached the boundary yet. Our results hold if $\overline{\mathcal{S}}(t)$ is a convex set or a finite union of convex sets. For simplicity reasons, we proceed as if $\overline{\mathcal{S}}(t)$ was made of a single convex set. In the sequel, and for simplicity reasons we shall omit the time index in $\underline{\mathcal{S}}(t)$ and $\overline{\mathcal{S}}(t)$. Figure 1 shows how $\mathcal{S}$ would be split in $\underline{\mathcal{S}}$ and $\overline{\mathcal{S}}$ by two frontiers $\theta_{1}$ and $\theta_{2}$. Note that the right panel is just a linear representation of the circle, which may be helpful in the numerical exercises. 


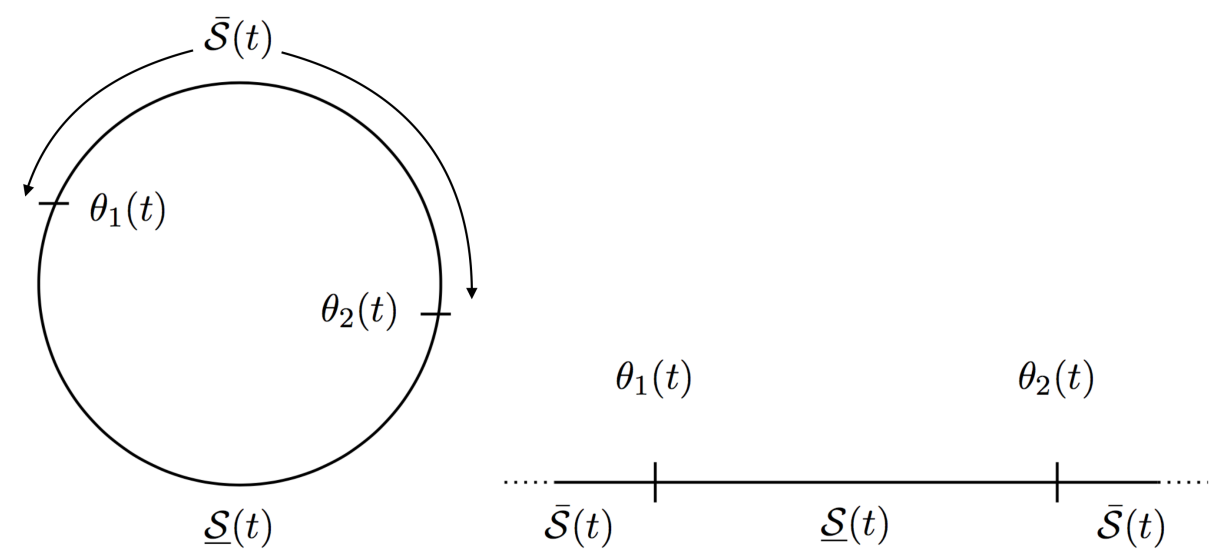

Figure 1: Description of the pollution frontier.

In Physics, the Stefan equation describes the evolution in time of the frontier between two phases of a matter. Typically, it has been applied to describe the frontier between the solid and liquid phases of water. ${ }^{9}$ In our problem, we assume that the pollution frontier delimiting the fertile region $\overline{\mathcal{S}}$ moves according to the Stefan equation, following the spatial gradient of polluted land. If at time $t \geq 0, \underline{\mathcal{S}}(t)=\left[\theta_{1}(t), \theta_{2}(t)\right] \subset \mathcal{S}$, then

$$
\dot{\theta}_{1}(t)=-D \frac{\partial L_{P}\left(t, \theta_{1}\right)}{\partial \theta}=D \frac{\partial L_{F}\left(t, \theta_{1}\right)}{\partial \theta} \quad \text { and } \quad \dot{\theta}_{2}(t)=-D \frac{\partial L_{P}\left(t, \theta_{2}\right)}{\partial \theta}=D \frac{\partial L_{F}\left(t, \theta_{2}\right)}{\partial \theta} .
$$

Figure 2 below describes graphically the dynamics of the pollution frontier.

$$
\begin{gathered}
L_{F}(t, \theta)=L(\theta) \underset{\theta_{1}(t)}{\leftarrow} L_{F}(t, \theta)<L(\theta) \underset{\theta_{1}}{\stackrel{\theta_{2}(t)}{\rightarrow}} L_{F}(t, \theta)=L(\theta) \\
\dot{\theta}_{1}(t)=L_{F, \theta}\left(t, \theta_{1}\right)<0 \quad \dot{\theta}_{2}(t)=L_{F, \theta}\left(t, \theta_{1}\right)>0
\end{gathered}
$$

Figure 2: Dynamic pollution frontier

We provide next the optimal consumption policy in this Phase 2 and the optimal dynamics for fertile soil in the polluted region.

Proposition 4. Suppose that at least one location reaches the upper bound for fertile soil at time $\tau \geq 0$ and that Assumptions 1 and 2 hold. If at time $t \geq \tau, \theta \in \overline{\mathcal{S}}(t)$, then $L_{F}^{*}(t, \theta)=L(\theta)$

\footnotetext{
${ }^{9}$ The Stefan condition is expressed as a law of energy conservation, and in the case of water, the moving frontier depends on the change of temperature. Hence, if $u(x, t)$ denotes the temperature of water at location $x$ in a space $X$ at a given time $t \geq 0$, and $\xi(t)$ is the frontier between solid and liquid water, then

$$
\eta \rho \dot{\xi}(t)=c_{1} \frac{\partial u\left(x^{-}, t\right)}{\partial x}-c_{2} \frac{\partial u\left(x^{+}, t\right)}{\partial x},
$$

where $\xi(0)=0$, so that initially all space is frozen, and water melts when temperature attains an upper bound, that is $u(x, t) \geq U$ for some $U \in \mathbb{R}$. $c_{1}$ and $c_{2}$ are the coefficients of heat conductivity, $\rho$ is the density of the solid phase and $\eta$ is the latent heat of melting per unit of mass.
} 
and its optimal consumption is

$$
c^{*}(t, \theta)=A(\theta) \frac{\phi(\theta)-\nu(\theta)}{\phi(\theta)} \frac{L(\theta)}{N(\theta)}, \text { for all } t \geq \tau .
$$

If on the contrary, $\theta \in \underline{\mathcal{S}}(t)$, then optimal consumption can be expressed as

$$
c^{*}(t, \theta)=\left(\phi \beta_{0} e_{0}\right)^{-\frac{1}{\sigma}} \int_{\underline{\mathcal{S}}(t)} L_{F}^{*}(t, z) \beta_{0} e_{0}(z) d z,
$$

where $e_{0}$ is the first eigenvector of the problem $\mathcal{L} u=\lambda u$ on $\mathcal{S} . \beta_{0}$ is the following function of time

$$
\beta_{0}^{\frac{1-\sigma}{\sigma}}=\frac{\sigma}{\rho-\lambda_{0}(1-\sigma)} \int_{\underline{\mathcal{S}}(t)}\left[\phi(z) e_{0}(z)\right]^{-\frac{1-\sigma}{\sigma}} N(z) d z,
$$

and the optimal distribution of fertile soil in $\underline{\mathcal{S}}$ is a solution to the integro-differential equation

$$
\begin{aligned}
\int_{\underline{\mathcal{S}}(t)} L_{F}^{*}(t, z) e_{0}(z) d z & =\int_{\underline{\mathcal{S}}(t)} L_{F}^{*}(\tau, z) e_{0}(z) d z e^{\frac{\lambda_{0}-\rho}{\sigma}(t-\tau)} \\
& +\int_{\tau}^{t} e^{\frac{\lambda_{0}-\rho}{\sigma}(t-s)}\left[L\left(\theta_{2}(s)\right) e_{0}(2 \pi) \dot{\theta}_{2}(s)-L\left(\theta_{1}(s)\right) e_{0}(0) \dot{\theta}_{1}(s)\right] d s .
\end{aligned}
$$

The dynamics of the pollution frontier $\left[\theta_{1}(t), \theta_{2}(t)\right]$ is described by the differential equations

$$
\dot{\theta}_{1}(t)=D \frac{\partial L_{F}^{*}\left(t, \theta_{1}\right)}{\partial \theta} \text { and } \dot{\theta}_{2}(t)=D \frac{\partial L_{F}^{*}\left(t, \theta_{2}\right)}{\partial \theta},
$$

together with the initial condition $\left(\theta_{1}(\tau), \theta_{2}(\tau)\right)$.

Contrary to Phase 1, consumption in the polluted region $\underline{\mathcal{S}}$ does not grow at a constant rate. Among other things, optimal consumption depends on the evolution of the pollution frontier and on the abatement efficiency $\phi$. In contrast, consumption in the fertile region $\overline{\mathcal{S}}$ is constant in time, although heterogeneous in space. More productive locations, those less populated and those with a higher total land endowment consume more. Moreover, optimal consumption in $\overline{\mathcal{S}}$ ensures that each location abates its own pollution, i.e. $\phi\left(A L-c^{*} N\right)=$ $\nu A L$, avoiding this way the diffusion of pollution to its neighbors. By the same token, the fertile region does no longer contribute to the abatement of overall pollution.

Regarding the long-term in Phase 2, the following proposition shows that either all locations become fertile or an ever-polluted region will emerge:

Proposition 5. In Phase 2, the economy can reach a steady state with two distinct regions constant in size, $\mathcal{S}=\overline{\mathcal{S}} \cup \underline{\mathcal{S}}$, separated by a fixed frontier $\left\{\bar{\theta}_{1}, \bar{\theta}_{2}\right\}$. In $\overline{\mathcal{S}}$ fertile soil equals total land and optimal consumption is given by (7). The steady state for fertile soil in region $\underline{\mathcal{S}}$, $\bar{L}_{F}$, is a solution to $D \bar{L}_{F}^{\prime \prime}+A(\phi-\nu) \bar{L}_{F}=0$, with $L_{F}\left(\bar{\theta}_{1}\right)=L\left(\bar{\theta}_{1}\right)$ and $L_{F}\left(\bar{\theta}_{2}\right)=L\left(\bar{\theta}_{2}\right)$.

Proposition 5 presents one of the main findings of the paper: a polluted region $\underline{\mathcal{S}}$ may 
fall in an environmental poverty trap and never catch up with the fertile region. This result may come as a surprise since it shows the existence of an heterogeneous steady state even in an homogeneous economy with diffusion. Let us describe one of the possibly many genesis of an heterogeneous steady state: When the most advanced locations become fully fertile, they stop absorbing others' pollution. From that moment onwards still polluted locations face an increased challenge since they will have to satisfy consumers' demand with their inferior land endowment but without the external help of the leader region. The polluted region may fall into an environmental poverty trap if its production or abatement technologies are not advanced enough to cope with their own pollution plus, probably, some additional pollution from their neighbors.

\section{Numerical experiments}

The previous section has provided with analytical solutions for optimal fertile soil and consumption in the two phases of the economy. In this section, we develop numerical exercises to shed light on some of the remaining open questions. In particular, Subsection 4.1 explores the dependency of the optimal solution on the spatial heterogeneity of some of the model components during Phase 1. Set in Phase 2, Subsection 4.2 proposes different exercises that describe the dynamics of the optimal solution and the evolution of the pollution frontier. We first study the convergence towards the fertile economy. Then, we study the resiliency of two technologically apart fertile economies to a pollution shock. ${ }^{10}$

Regarding the model's calibration, the discount rate $\rho$ is set to $3 \%$ as in Boucekkine et al. (2018) and Lopez (2008). Using micro-household data, Attanasio and Weber (1993) and Attanasio and Browning (1995) show that $\sigma$ lies in an interval between 1.25 and 3.33. Here we assume that $\sigma=2$ following Barro et al. (1995). The amount of available land is 10 at all locations. Population is uniformly distributed, with $N(t, \theta)=1$ for all $t$ and $\theta$. Finally, we set the diffusion parameter $D$ to 1 .

\subsection{Optimal solution in Phase 1}

We study the detrended optimal consumption distribution $c_{g}^{*}(\theta)=e^{-g t} c^{*}(t, \theta)$ and then for the dynamics of fertile soil for two values of the abatement efficiency, $\phi$, and different functional forms for the soil sensitivity to pollution, $\nu$.

\footnotetext{
${ }^{10}$ Subsection 4.1 uses the package Chebfun in Matlab. An algorithm has been developed in Matlab to simulate the optimal solution in Phase 2.
} 
The role of abatement efficiency $\phi$ in inequality. Let us compare two economies with a technological pole, which only differ in their abatement efficiency. In particular, suppose there exists a technological pole around $\pi$, and let us analyze the effect of the abatement efficiency when $\phi=2$ and $\phi=3$. Figure 4 shows the given spatial distributions of productivity $A$, abatement efficiency $\phi$, soil sensitivity $\nu$ and the resulting trajectories for optimal detrended consumption $c_{g}^{*}$.
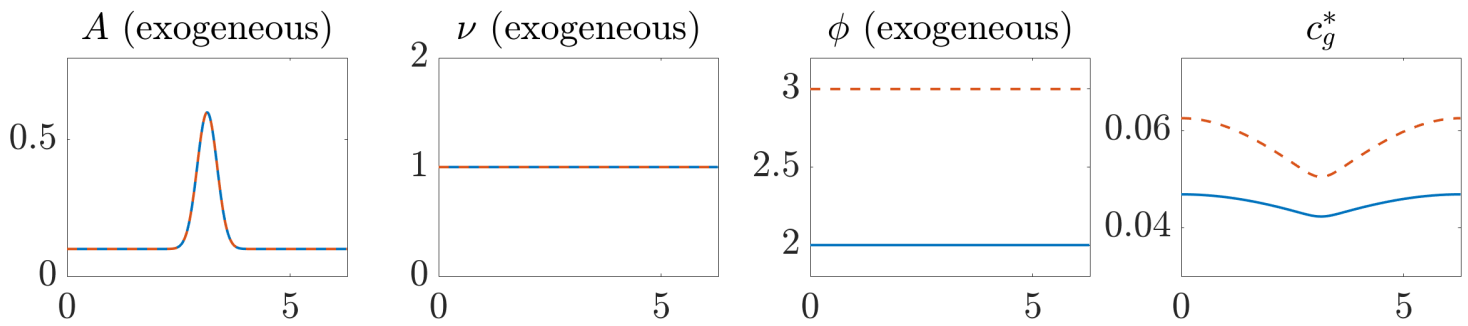

Figure 3: Impact of $\phi$ on $c_{g}^{*}$ in Phase 1. Dashed lines correspond to $\phi=3$; solid lines to $\phi=2$.

Since net productivity $A(\phi-\nu)$ increases with $\phi$, optimal consumption also increases with $\phi$ at each location. Further, Figure 3 shows that consumption is heterogeneous and that consumption inequality increases with abatement efficiency. Surprisingly, consumption is lower in the technologically advanced locations. There are two reasons behind this behavior. First and independently of $\phi$, pollution damage $\nu A L_{F}$ is stronger in the technological pole. As a result, the social planner sacrifices consumption there in order to abate the local excess in pollution. Second, the central area can abate more efficiently since $A \phi$ is also larger. For this reason the policy maker induces these locations to abate even further to act as a pollution sink. In doing so, consumption diminishes further and fosters heterogeneity.
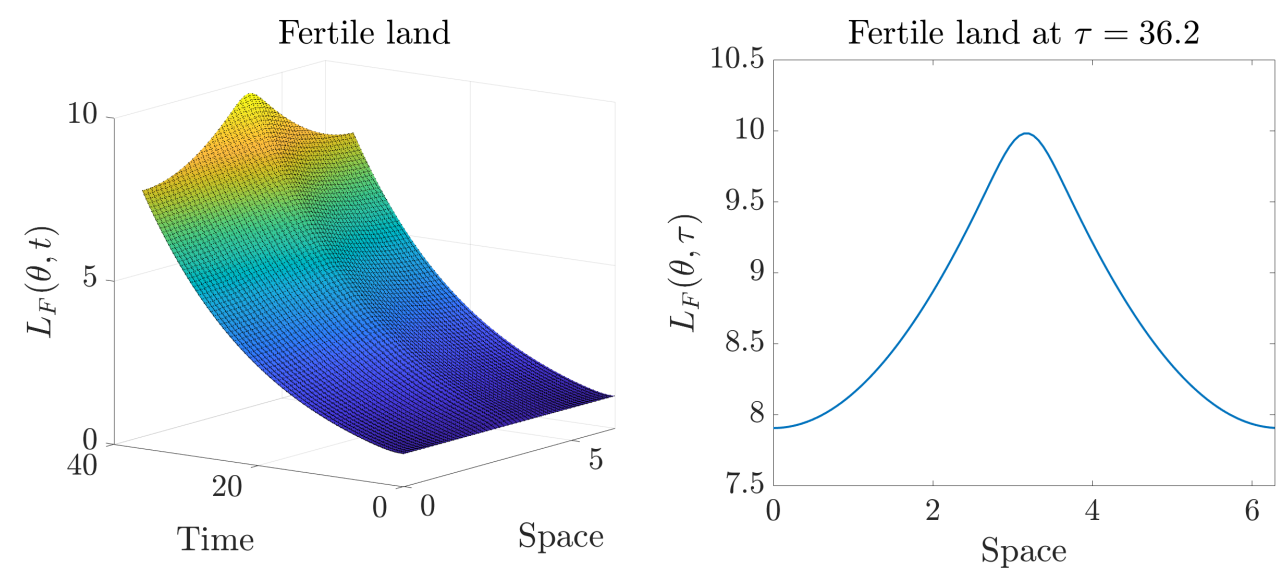

Figure 4: Dynamics of fertile soil in Phase 1 for $\phi=2$ starting with $L_{F}^{0}(\theta)=1$ for all $\theta \in \mathcal{S}$.

With time, the technological pole accumulates faster fertile soil and they are first to reach their fertility upper bound (see Figure 4). Moreover, the advantage of the core region over the 
periphery gets even more pronounced. Figure 5 shows that the more efficient the abatement technology, the more unequal the economy (in terms of $L_{F}$ ). A higher $\phi$ also reduces the length of Phase 1, which, as we will later see may irremediable break appart the economy, segregating fertile from non fertile locations forever.

Proposition 1 proves that during Phase $1 g$ is constant in time and spatially homogeneous. Our numerical exercises additionally show that $g$ increases with $\phi$. Indeed, $g$ grows from $6 \%$ to $14.1 \%$ when $\phi$ increases from 2 to 3 . That is, an increase of $50 \%$ in the abatement efficiency implies an increase of $135 \%$ in the growth rate, pushing the economy towards Phase 2 at a radically faster pace. Indeed, when $\phi=2$ the economy reaches Phase 2 in 36.2 units of time, i.e. $\tau=36.2$, while $\tau=14.4$ when $\phi=3$.

This exercise has revealed the hidden consequences of improving abatement. Good abators will produce, consume and abate better, act as pollution sinks. Obviously, inequality between good and bad abators widens. Land also becomes fertile at a faster rate which will eventually push the economy faster into Phase 2 and trigger inequality even further since the good abators will stop absorbing others' pollution.

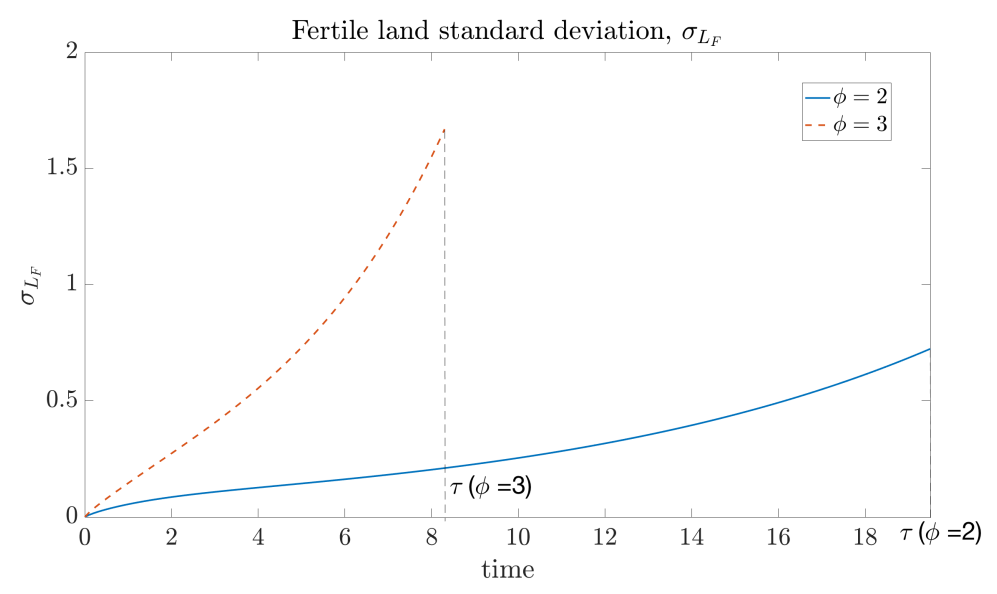

Figure 5: $\sigma_{L_{F}}$ as a function of time for $\phi=2$ and $\phi=3$.

On technology, sensitivity and resilience. Suppose that efficient technologies cause greater damages to soil, locally eroding the area's resilience. The dependence of soil sensitivity to productivity is very simply modeled using a power function: $\nu=\nu_{0} A^{\gamma}$, with $\nu_{0} \in \mathbb{R}$ and $0<\gamma<1$. To make sure we control all sources of heterogeneity, we let vary abatement efficiency and consider two different values for $\phi$, namely $\phi=10$ and $\phi=11$. 

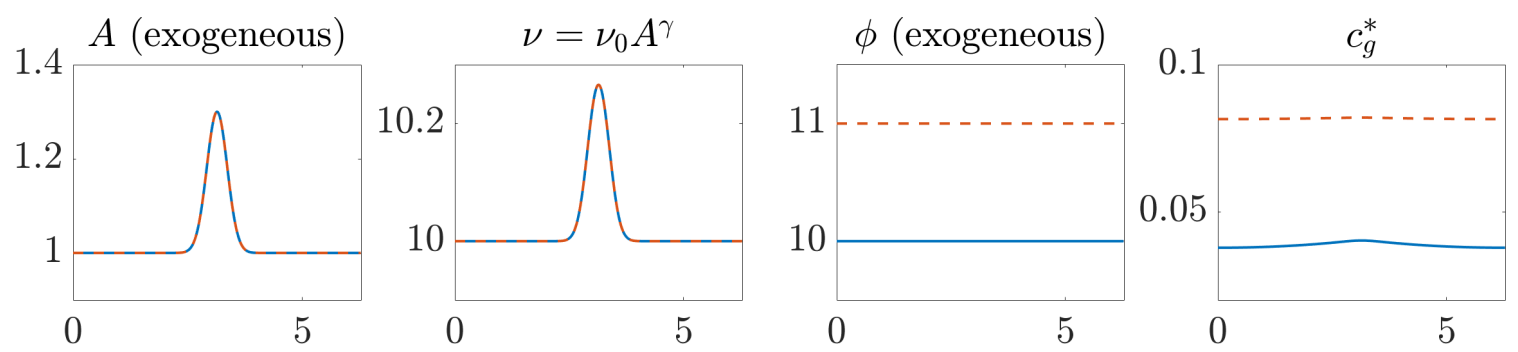

Figure 6: Impact of $\phi$ on $c_{g}^{*}$ with spatially heterogeneous $\nu$. Dashed lines correspond to $\phi=11$; solid lines to $\phi=10$.

Figure 6 shows that as abatement efficiency increases, detrended consumption increases as in the previous example. This time the increase in consumption comes at the expense of further pollution, which cannot be absorbed despite the advanced technology. The situation of the technological pole worsens further because the area is highly sensitive to pollution. As a result, fertile land will lag behind in the technological pole and the first locations to become fully fertile are in the periphery, despite having the least productive and least damaging technology.

Obviously, this pattern hinges on $\phi$. If $\phi$ is very large, then all pollution is absorbed and the technologicall advanced region willb e first to reach Phase 2.
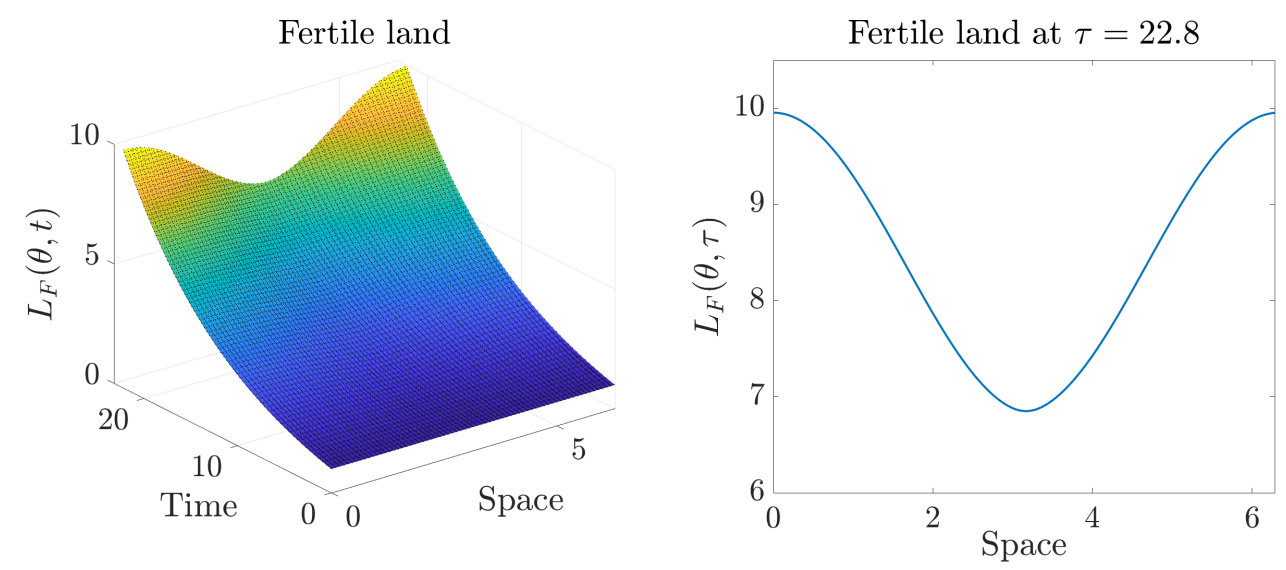

Figure 7: Dynamics of fertile soil in Phase 1 for $\phi=11$ starting with $L_{F}^{0}=1$ for all $\theta$.

\subsection{Fertile soil dynamics in Phase 2}

Let us explore numerically Phase 2. The first exercise continues the first example of section 4.1, describing the dynamics of fertile soil from the moment Phase 2 is reached. We will learn that optimal dynamics and convergence are far from monotonic or balanced growth paths. The second set of exercises investigates the resiliency of the fertile economy to pollution shocks and explores the existence of a spatially heterogeneous steady state.

When technology is constant we choose $A=0.2$, smaller than the standard value of 1 , to 
underline that the economy is agricultural. In the cases where $A$ is spatially heterogeneous, $A$ will be an exponential function. Regarding the remaining parameters, $\phi$ is equal to $2, \nu$ is 0.2 and population is homogeneous and equal to 10 .

Convergence towards the fertile economy. This exercise presents the continuation of the optimal solution studied in Subsection 4.1 and depicted in Figure 4, in which the central region attains Phase 2 at $\tau=36.2$. From that moment onwards, locations at the maximum of fertile soil only abate their own pollution to safeguard their fertile land, and diffusion only takes place in the polluted region and across the frontier. That is, locations with the largest amount of fertile soil stop absorbing pollution from neighboring locations. As Figure 8 shows, this change seems to come as a shock for all partially polluted locations, where pollution instantaneously rises. Let us underline that the policy maker had obviously foreseen the passage from Phase 1 to Phase 2, and it is by no means a shock, it is actually an optimal adaptation pattern. Figure 8 shows how at locations around $\theta=0$ (or $\theta=2 \pi$ ), fertile soil drops from 7.9 to 7.4 at the moment Phase 2 is hit. What happens later is that abatement rates increase in $\underline{\mathcal{S}}$ and fertile soil starts growing again so that the polluted region starts catching up. The right panel in Figure 8 shows the growth rate of fertile soil. Note that in contrast to Phase 1, the growth rate is not in the least constant in time nor spatially homogeneous. Locations where fertile soil had dropped in the most severe manner grow the fastest later on.
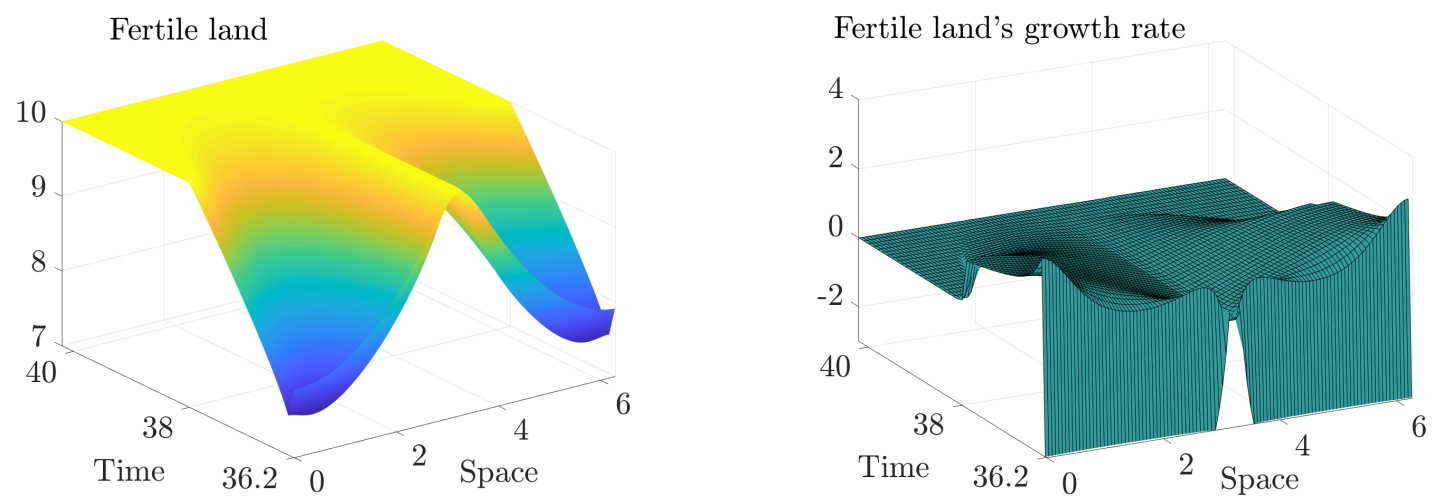

Figure 8: Dynamics in Phase 2 towards the fertile economy for $\phi=2$.

Resiliency of the fertile economy to pollution shocks and the emergence of heterogeneous steady states. In this last exercise, the central region of a fully fertile economy receives a pollution shock. In particular, the central region $\left[\frac{4.5}{10} 2 \pi, \frac{5.5}{10} 2 \pi\right]$ of size 1 receives a pollution shock, which induces a loss of $1 \%$ of its fertile soil, that is, $L_{F}(0, \theta)=0.99 L(\theta)$ for $\theta \in\left[\frac{4.5}{10} 2 \pi, \frac{5.5}{10} 2 \pi\right]$. In order to seize the major role played by technology, two different 
distributions are considered a homogeneous distribution with $A=0.2$ and on heterogeneous distribution $A(\theta)=0.20+2 e^{-(\theta-\pi)^{2}}$, which generates a technological pole in the middle area.

Several interesting features will come to light, first we conclude that a pollution shock can be overcome only if the region under ecological stress is endowed with a sufficiently advanced technology. Second, after-shock changes in the economy may come in long stages, which may seem wrongfully definitive if the time span we consider is not long enough. Indeed, if attention is focused on the fertile region and its frontier, forgetting the latent stock of pollution in the polluted region, then agents will not foresee the second pollution wave. A second wave does arise when the polluted region attains its maximum abatement and resilience capacity.
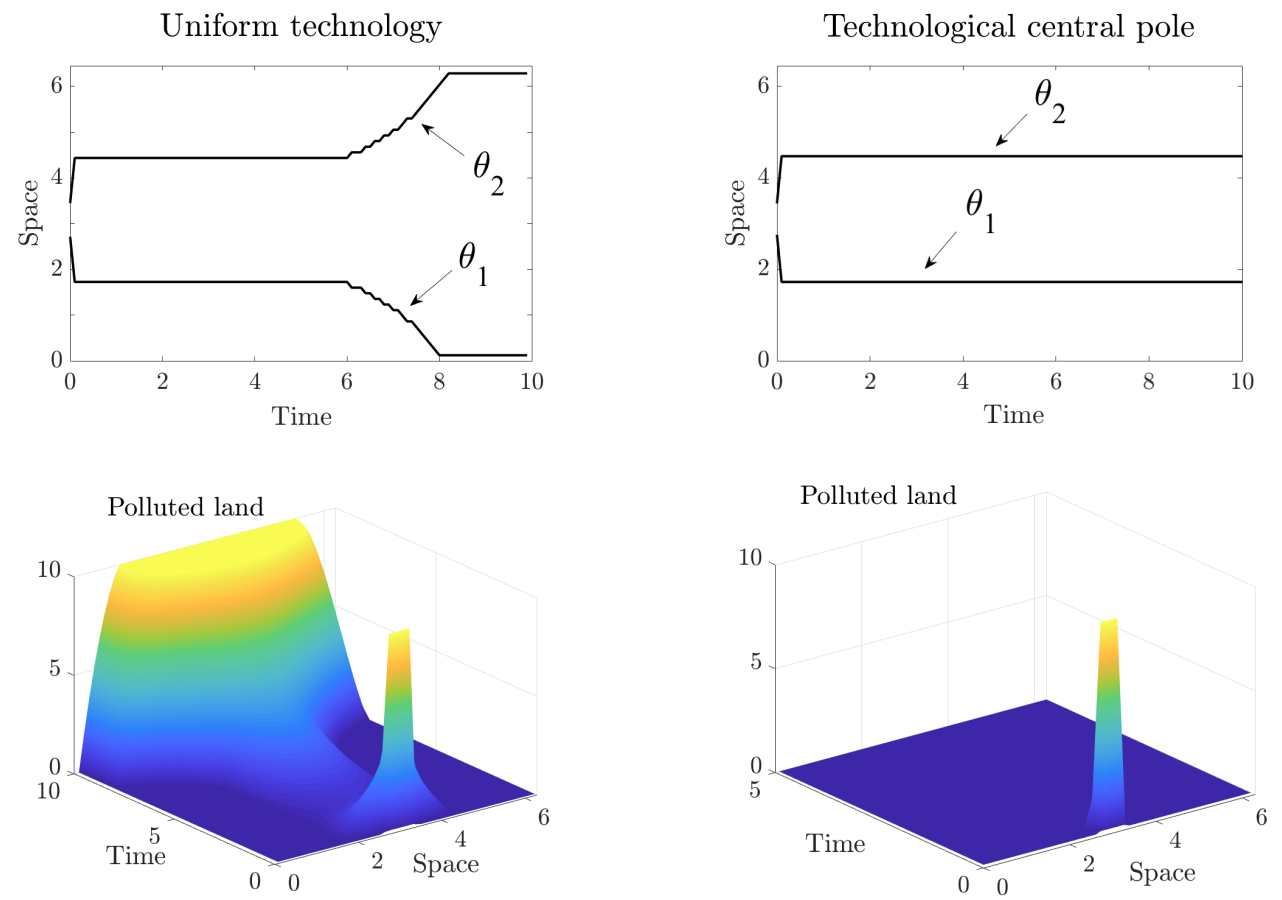

Figure 9: Spatial dynamics for initial shock $L_{F}(0, \theta)=0.99 L(\theta)$ for $\theta \in\left[\frac{4.5}{10} 2 \pi, \frac{5.5}{10} 2 \pi\right]$.

Let us analyze the different stages the economy goes through after receiving a pollution shock (see Figure 9). Right after the shock, polluted land increases, and this is independent on technology. Indeed, the remaining fertile soil has to face now a large stock of pollution, and in order to preserve consumption in as much as possible, abatement will be sacrified temporarily. Additionally, once pollution is present in the soil, it accumulates in time. As a result, after the initial shock, polluted land rockets.

Let us follow the evolution of the economy when technology is homogeneous (left column of Figure 9). Right after the shock, pollution diffuses over space pushing the frontiers of the polluted region. As the top-left graph in Figure 9 shows, the polluted region becomes stable in size for some time, and the level of pollution lowers. Nevertheless, this situation does not last. Indeed, the fall in polluted land is followed by a very slow and subtle accumulation 
of pollution at all polluted locations. Once a threshold of pollution is reached, the region is not able to abate enough. As a result, pollution starts diffusing again, pushing further the frontiers of the polluted region. At that moment there is a massive uprise in pollution everywhere. Indeed, not only local pollution accumulates due to production, but previously protected locations start receiving pollution from neighboring locations. Again, locations tend to preserve consumption, diminishing abatement, which makes pollution increase even more. Noteworthy, in this example, locations at the maximum of fertile soil at the beginning, and which do not abate more than needed, end up being highly polluted by the diffusion mechanism.

In sharp contrast, when the shocked region has a technological advantage, locations can overcome the shock by adapting their consumption and abatement during the initial moments (right column in Figure 9). Although polluted land increases at first, note that the central locations are more productive and can produce more using less land and polluting less. This mechanism counterbalances the shock, explaining why the central region absorbs all pollution in an extremely short period of time.

This exercise has illustrated the existence of non homogeneous steady states, even when all the model's parameters are spatially homogeneous. We have also learnt about their emergence, following the changes in the pollution frontier and revealing recovery thresholds in technology.

\section{Conclusion}

This paper has developed a spatial growth model accounting for the diffusion of pollution in soils, and it has provided with the explicit optimal trajectory for fertile soil and consumption. We have faced two major challenges. First, the production factor, fertile soil, is naturally bounded by the amount of available land at each location. Second, at any moment in time the economy can be made of a polluted and a fertile region separated by a frontier. Since pollution diffuses, the frontier can actually continuously change with time. Borrowing the Stefan's equation from Physics, we have modeled the dynamics of the pollution frontier around the fertile region.

The boundedness of the production factor defines two phases in the dynamics of fertile soil. In the first phase there is some polluted land everywhere, and fertile soil and consumption grow at the same constant rate at all locations. Eventually, some locations eradicate all pollution and the economy enters the second phase. Here the dynamics change radically. Locations at the maximum stop absorbing pollution coming from the polluted area. Different long-term patterns can emerge. Eventually, the polluted region may actually never reach its 
potential maximum for fertile soil, and remain forever in a sort of environmental poverty trap. This possibility has been analytically proven and numerically explored.

Although we did not develop any policy recommendations, this paper has shown that specially after a pollution shock, policy makers should leave temporarily aside their optimal policies which discount heavily the far future and induce instead fertile regions to help reduce overall pollution. Refusing to do so could optimally lead to an entirely polluted economy.

\section{References}

[1] Augeraud-Véron, E., Boucekkine, R. and Veliov, V. (2019), "Distributed optimal control models in environmental economics: a review", Mathematical Modelling of Natural Phenomena. 14, 106.

[2] Antle, J. M., Stoorvogel, J. J., Valdivia, R. O. (2006), "Multiple equilibria, soil conservation investments, and the resilience of agricultural systems", Environ. Dev. Econ., 11(4), 477-492.

[3] Attanasio, O. and Browning, M. (1995), "Consumption over the Life Cycle and over the Business Cycle", American Economic Review, 85(5), 1118-1137.

[4] Attanasio, O. and Weber, G. (1993), "Consumption, the Interest Rate and Aggregation", Review of Economic Studies, 60(3), 631-649.

[5] Ballestra, V. L. (2016), "The spatial AK model and the Pontryagin maximum principle", Journal of Mathematical Economics, 67, 87-94.

[6] Barbier, E. B. (1990), "The farm-level economics of soil conservation: the uplands of Java", Land Econ., 66(2), 199-211.

[7] Barrett, S. (1991), "Optimal soil conservation and the reform of agricultural pricing policies", $J$. Dev. Econ., 36(2), 167-187.

[8] Barrett, C. B. and Bevis, L. E. (2015), "The self-reinforcing feedback between low soil fertility and chronic poverty", Nat. Geosci., 8(12), 907-912.

[9] Barro R., Mankiw, G. and Sala-i-Martin, X. (1995), "Mobility in Neoclassical models of Growth", American Economic Review, 85(1), 103-115.

[10] Bensoussan, A., Da Prato, G., Delfour, M. C. and Mitter, S. K. (2007), "Representation and control of infinite dimensional system", Second edition, Birkhauser, Boston.

[11] Berazneva, J., Conrad, J., Gerena, D., Lehmann, J. and Woolf, D. (2018), "Agricultural productivity and soil carbon dynamics: a bioeconomic model", Working Paper.

[12] Bevis, L. E., Conrad, J. M., Barrett, C. B. and Gray, C. (2017), "State-conditioned soil investment in rural Uganda", Res. Econ., 71(2), 254-281.

[13] Boucekkine, R., Camacho, C. and Zou, B. (2009), "Bridging the gap between growth theory and the new economic geography: The spatial Ramsey model", Macroeconomic Dynamics, 13, 20-45.

[14] Boucekkine, R., Camacho, C. and Fabbri, G. (2013), "Spatial dynamics and convergence: The spatial AK model", Journal of Economic Theory, 148, 2719-2736. 
[15] Boucekkine, R., Fabbri, G., Federico, S. and Gozzi, F. (2018), "Growth and agglomeration in the heterogeneous space: a generalized AK approach", Journal of Economic Geography, 0, 1-32.

[16] Boucekkine, R., Fabbri, G., Federico, S. and Gozzi, F. (2020), "From firm to global-level pollution control: the case of transboundary pollution", Quaderni del Dipartimentodi Economia Politica e Statistica.

[17] Brock, W., Xepapadeas, A. (2008), "Diffusion-induced instability and pattern formation in infinite horizon recursive optimal control", J. Econ. Dyn. Control, 32, 2745-2787.

[18] Brock, W., Xepapadeas, A. (2010), "Pattern formation, spatial externalities and regulation in coupled economic-ecological systems", J. Environ. Econ. Manag., 59, 149-164.

[19] Bunce, Arthur C. (1974), "Economics of Soil Conservation", Ames: Iowa State University Press.

[20] Burt, O.R. (1981), "Farm level economics of soil conservation in the Palouse area of the Northwest", Am. J. Agric. Econ., 63(1), 83-92.

[21] Camacho, C. and Pérez-Barahona A. (2016), "Land use dynamics and the environment", Journal of Economic Dynamics and Control, 52, 96-118.

[22] CCICED (2015), CCICED 2015 Work Report.

[23] Chartier, P.M. and Rostagno, C.M. (2006), "Soil Erosion Thresholds and Alternative States in Northeastern Patagonian Rangelands", Rangeland Ecology and Management, 59(6), 616-624.

[24] Ciriacy-Wantrup, S. V. (1968), "Resource Conservation-Economics and Policies", Berkeley: University of California Agr. Exp. Sta.

[25] Coddington, E. A., Levinson, N. (1955), "Theory of Ordinary Differential Equations", New York: McGraw-Hill.

[26] Dupouey, J.J., Dambrine, E., Laffite, J.D. and Moares, C. (2002), "Irreversible Impact of Past Land Use on Forest Soils and Biodiversity", Ecology, 83(11), 2978-2984.

[27] EU Commission (1991), Directive 91/676/EEC. "Council Directive of 12 December 1991 concerning the protection of waters against pollution caused by nitrates from agricultural sources", Official Journal of European Community L375: 1-8.

[28] FAO and ITPS. 2015. Status of the WorldÕs Soil Resources (SWSR) Technical Summary. Food and Agriculture Organization of the United Nationsand Intergovernmental Technical Panel on Soils, Rome, Italy.

[29] Food and Agriculture Organization of the United Nations and Intergovernmental Technical Panel on Soils Status (2015), Status of the World's Soil Resources Main Report.

[30] Gao, Y., Zhong, B., Yue, H., Wu, B. and Cao, S. (2011), "A degradation threshold for irreversible loss of soil productivity: a long?term case study in China", Journal of Applied Ecology, 48, 11451154.

[31] de Graaf, J., Amsalu, A., Bodnár, F., Kessler, A., Posthumus, H., Tenge, A. (2008), "Factors influencing adoption and continued use of long-term soil and water conservation measures in developing countries", Appl. Geography, 28(4), 271-280. 
[32] Hagos, F., Holden, S. (2006), "Tenure security, resource poverty, public programs, and household plot-level conservation investments in the highlands of northern Ethiopia", Agric. Econ., 34(2), 183-196.

[33] Kopittke, P.M., Menzies, W.N., Wang, P., McKenna, B.A. and Lombi, E. (2019), "Soil and the intensification of agriculture for global food security", Environment International, 132.

[34] La Torre, D., Liuzzi, D. and Marsiglio, S. (2015), "Pollution diffusion and abatement activities across space and over time", Mathematical Social Sciences, 78, 48-63.

[35] Le Kama, A.A., Pommeret, A. and Prieur, F. (2014), "Optimal Emission Policy under the Risk of Irreversible Pollution", Journal of Public Economic Theory, 16(6), 959-980.

[36] Lopez, H. (2008), "The social discount rate : estimates for nine Latin American countries", The World Bank Policy Research Working Paper Series 4639.

[37] McConnell, K.E. (1983), "An economic model of soil conservation", Am. J. Agric. Econ., 65(1), 83-89.

[38] Pope, C.A., Bhide, S., Heady, E.O. (1983), "The economics of soil conservation: an optimal control theory approach", North Central J. Agric. Econ., 5(2), 83-89.

[39] Saliba, B.C. (1985), "Soil productivity and farmers' erosion control incentives? A dynamic modeling approach", Western J. Agric. Econ., 10(2), 354-364.

[40] Segarra, E., Taylor, D.B. (1987), "Farm level dynamic analysis of soil conservation: an application to the piedmont area of Virginia", J. Agric. Appl. Econ., 19(2), 61-73.

[41] Smith, M., Sanchirico, J., Wilen, J. (2009), "The economics of spatial-dynamic processes: applications to renewable resources", J. Environ. Econ. Manag., 57, 104-121.

[42] Stephens, E. C., Nicholson, C. F., Brown, D. R., et al. (2012), "Modeling the impact of natural resource-based poverty traps on food security in Kenya: The Crops, Livestock and Soils in Smallholder Economic Systems (CLASSES) model", Food Security, 4(3), 423-439.

[43] "United Nations Convention to Combat Desertification", (2017), The Global Land Outlook, first edition.

\section{Appendices}

\section{A. Mathematical Preliminaries}

We represent (2) as an abstract dynamical system in infinite dimension. ${ }^{11}$ Its solutions belong to $L^{2}(\mathcal{S})$, defined as

$$
L^{2}(\mathcal{S})=\left\{f:\left.\mathcal{S} \longrightarrow \mathbb{R}\left|\int_{0}^{2 \pi}\right| f(\theta)\right|^{2} d \theta<\infty\right\}
$$

\footnotetext{
${ }^{11}$ A similar account can be found in Boucekkine et al. (2013). For a deeper insight see Bensoussan et al. (2007).
} 
For any two functions $f, g$ in $L^{2}(\mathcal{S})$, their inner product is defined as $\langle f, g\rangle=\int_{0}^{2 \pi} f(\theta) g(\theta) d \theta$, and $f$ 's norm is then $|f|^{2}=<f, f>=\int_{0}^{2 \pi} f^{2}(\theta) d \theta . L^{2}(\mathcal{S})$ endowed with the inner product $\langle\cdot, \cdot>$ is a Hilbert space. Since we cannot take derivatives on all elements of $L^{2}(\mathcal{S})$, let us define three spaces that will help us defining the domain of $\mathcal{L}$

$$
\begin{aligned}
\mathcal{H}_{+}^{0}(\mathcal{S}) & :=\left\{f \in L^{2}(\mathcal{S}): f(\cdot) \geq 0, \text { and } f(\cdot) \neq 0\right\} \\
\mathcal{H}^{1}(\mathcal{S}) & :=\left\{f \in L^{2}(\mathcal{S}): \exists \text { f' in weak sense and belongs to } L^{2}(\mathcal{S})\right\} \\
\mathcal{H}^{2}(\mathcal{S}) & :=\left\{f \in L^{2}(\mathcal{S}): \exists \text { f' in weak sense and belongs to } \mathcal{H}^{1}(\mathcal{S})\right\}
\end{aligned}
$$

Hence, the domain of $\mathcal{L}$ is $\mathcal{H}^{2}(\mathcal{S})$ and $\mathcal{L}$ is well defined on this domain. Operator $\mathcal{L}$ is self-adjoint since for any two $\left.f, g \in \mathcal{H}^{2}(\mathcal{S}),\langle\mathcal{L} f, g\rangle=<f, \mathcal{L}^{*} g\right\rangle=<f, \mathcal{L} g>$. Note first that the Laplacian operator is closed in its domain, $\mathcal{H}^{2}(\mathcal{S})$, and it generates a semigroup on $L^{2}(\mathcal{S})$. Since the second factor in $\mathcal{L}$ is bounded, we can conclude that our operator $\mathcal{L}$ is closed in its domain, $D(\mathcal{L})=\mathcal{H}^{2}(\mathcal{S})$ and it generates a $C_{0}$ semigroup.

The Laplacian operator generates a $C_{0}$ semigroup in the following sense. Let $\mathcal{A}$ be a closed linear operator defined on $L^{2}(\mathcal{S})$ (for simplicity), and consider the following initial value problem

$$
\begin{aligned}
y^{\prime}(t) & =\mathcal{A} y(t) \\
y(0) & =y_{0} \in L^{2}(\mathcal{S}), \text { given. }
\end{aligned}
$$

If the operator $\mathcal{A}$ is bounded, then the solution to this problem is given by

$$
y(t)=e^{t \mathcal{A}} y_{0}:=\sum_{j=0}^{\infty} \frac{t^{j} \mathcal{A}^{j} y_{0}}{j !} .
$$

Let us define $Z_{\mathcal{A}}(t)=e^{t \mathcal{A}}$, then $Z_{\mathcal{A}}(t+s)=Z_{\mathcal{A}}(t) Z_{\mathcal{A}}(s)$, and $Z_{\mathcal{A}}(0)=1$. As mentioned in p. 89 in Bensoussan (2007), there is a one-to-one correspondence between the group $(\mathbb{R},+)$ and the subset $Z_{\mathcal{A}}=\left\{Z_{\mathcal{A}}(t): t \in \mathbb{R}\right\}$ in $L(\mathcal{S})$, or in particular in $L^{2}(\mathcal{S})$ under composition "o". So we say that $\left(Z_{\mathcal{A}}, \circ\right)$ is a group. When $\mathcal{A}$ is unbounded like in the case of the Laplacian operator, then one can define $Z_{\mathcal{A}}$ only for $t \geq 0$ and $s \geq 0$ in general. We say then that $Z_{\mathcal{A}}$ is a semigroup.

The most useful property of semigroups hinges on the following relationship. The infinitesimal generator $\mathcal{A}$ of $Z$ is the linear operator in $\mathcal{S}$ defined by

$$
D(\mathcal{A})=\left\{x \in \mathcal{S}: \text { such that } \lim _{h \rightarrow 0^{+}} \frac{Z(h) x-x}{h} \text { exists }\right\}
$$

and $\mathcal{A} x=\lim _{h \rightarrow 0^{+}} \frac{Z(h) x-x}{h}$ for all $x \in D(\mathcal{A})$. Then, we have that

$$
\frac{d}{d t} Z(t) x=\mathcal{A} Z(t) x=Z(t) \mathcal{A} x
$$

Given the set of admissible trajectories for $L_{F}$, we can define the set of admissible strategies for $c, \mathcal{C}$ as

$$
\mathcal{C}\left(L_{F}^{0}\right)=\left\{c \in \mathcal{L}_{\text {loc }}^{1}\left(\mathbb{R}^{t}, L^{2}(\mathcal{S})^{+}\right): L_{F} \in \mathcal{H}_{0}, \text { for all } t \geq 0\right\}
$$


Finally, the objective functional is

$$
J\left(L_{F}^{0} ; c\right):=\int_{0}^{\infty} e^{-\rho t} U(c) d t
$$

where

$$
U(c)=\int_{\mathcal{S}} \frac{c(t, \theta)^{1-\sigma}}{1-\sigma} N(\theta) d \theta .
$$

The value function associated to our problem is then

$$
V\left(L_{F}^{0}\right):=\sup _{c} J\left(L_{F}^{0} ; c\right)
$$

\section{B. Necessary optimal conditions}

Although our problem is close to Boucekkine et al. (2013) and Boucekkine et al. (2018), the production factor is here bounded. Most importantly, the policy maker needs to take into account the eventuality of reaching one of the boundaries, and that the economy could be split in two regions. The authors resort to an optimal control approach to sort this problem. Using Ekeland's variational principle, we first obtain the set of necessary conditions for our problem. Then we will go back and forth from optimal control to dynamic programming, being aware of the awkwardness it generates. As said earlier, the inclusion of a (moving) frontier and the possibility of two coexisting regions make us combine these two different approaches. We explain next all details.

The set of necessary optimal conditions obtained though the variational principle has the main shortcoming of including a reverse time parabolic partial differential equation, which makes our problem difficult to analyse. For this reason, we resort to dynamic programming to obtain the exact expression of the optimal solution of an auxiliary problem in which locations never reach their boundary. Fortunately, and since the optimal solution is unique (see Camacho and Pérez-Barahona, 2015), we can prove that the solution obtained through dynamic programming also satisfies the set of optimal conditions obtained via optimal control when fertile land lies within its boundaries. Then, we prove that during this first regime fertile land grows at a constant and spatially homogeneous rate, steadily increasing or decreasing with time. As a result, one location will eventually reach a boundary for fertile land. At that moment, the solution enters in the second phase. In this second phase, some of the locations have attained the maximum level of fertile land, while others are still transiting. Once locations reach the bound, they stop investing in pollution abatement. Let us see next all details.

In order to apply Ekeland's variational principle, let us write the value function $V(\cdot)$ associated to problem (2)-(5)

$$
\begin{gathered}
V\left(c, L_{F}, \psi, \mu\right)=\int_{0}^{\infty} \int_{0}^{2 \pi} e^{-\rho t} \frac{c(t, \theta)^{1-\sigma}}{1-\sigma} N(\theta) d \theta d t \\
-\int_{0}^{\infty} \int_{0}^{2 \pi} \psi(t)\left(\frac{\partial L_{F}}{\partial t}(t, \theta)-D \frac{\partial^{2} L_{F}}{\partial \theta^{2}}(t, \theta)-A(\theta)[\phi(\theta)-\nu(\theta)] L_{F}(t, \theta)+c(t, \theta) N(\theta) \phi(\theta)\right) d \theta d t \\
-\int_{0}^{\infty} \int_{0}^{2 \pi} \mu(t, \theta)\left[L(\theta)-L_{F}(t, \theta)\right] d \theta d t-\int_{0}^{\infty} \int_{0}^{2 \pi} \xi(t, \theta) L_{F}(t, \theta) d \theta d t .
\end{gathered}
$$

Then, assuming there exists an optimal solution and that any solution to our problem can be written 
as a deviation from the optimal solution we obtain

$$
\begin{array}{r}
c(t, \theta)=c^{*}(t, \theta)+\epsilon \mathcal{C}(t, \theta), \\
L_{F}(t, \theta)=L_{F}^{*}(t, \theta)+\epsilon l_{F}(t, \theta) .
\end{array}
$$

$V$ becomes then a function of $\epsilon$. At the optimal $\epsilon^{*}, \frac{\partial V\left(\epsilon^{*}\right)}{\partial \epsilon}=0$. We use that the auxiliary variables $\psi, \mu$ and $\xi$ are $\mathcal{C}^{1}$ functions, so that $\psi(t, 0)=\psi(t, \pi), \mu(t, 0)=\mu(t, \pi), \xi(t, 0)=\xi(t, \pi)$; and $\psi_{x}(t, 0)=$ $\psi_{x}(t, \pi), \mu_{x}(t, 0)=\mu_{x}(t, \pi), \xi_{x}(t, 0)=\xi_{x}(t, \pi)$. Since $L_{F} \in \mathcal{C}^{1}$, it is also true that $L_{F}(t, 0)=L_{F}(t, 2 \pi)$ and $L_{F, x}(t, 0)=L_{F, x}(t, 2 \pi)$. Then, since $V$ is continuously differentiable in $c$ and $L_{F}$, after applying integration by parts, we can compute $\frac{\partial V(\epsilon)}{\partial \epsilon}$ as

$$
\begin{gathered}
\frac{\partial V(\epsilon)}{\partial \epsilon}=\int_{0}^{\infty} \int_{0}^{2 \pi} e^{-\rho t} c(t, \theta)^{-\sigma} \mathcal{C}(t, \theta) N(\theta) d \theta d t \\
-\int_{0}^{\infty} \int_{0}^{2 \pi}\left[-\psi_{t}(t, \theta) \mathcal{L}_{F}(t, \theta)-D \psi_{x x}(t, \theta) l_{F}(t, \theta)\right] d \theta d t \\
-\int_{0}^{\infty} \int_{0}^{2 \pi}\left[A(\theta)[\phi(\theta)-\nu(\theta)] \psi(t, \theta) l_{F}(t, \theta)+\mathcal{C}(t, \theta) N(\theta) \phi(\theta)\right] d \theta d t \\
+\int_{0}^{\infty} \int_{0}^{2 \pi}[\mu(t, \theta)-\xi(t, \theta)] l_{F}(t, \theta) d \theta d t,
\end{gathered}
$$

with $\lim _{t \rightarrow \infty} \psi(t, \theta) L_{F}(t, \theta)=0$, for all $\theta \in \mathcal{S}$. Along with the transversality condition, we obtain the following condition on $L_{F}$ and $\psi$

$$
\left.\psi(t, \theta) \nabla_{\theta} L_{F}(t, \theta)\right|_{0} ^{2 \pi}=\left.\nabla_{\theta} \psi(t, \theta) L_{F}(t, \theta)\right|_{0} ^{2 \pi},
$$

where $\nabla_{\theta}$ is the space integral of $\mathcal{L}$.

Collecting the terms multiplying $\mathcal{C}$ and $l_{F}$, and time detrending $\psi$, we obtain the following set of necessary optimal conditions for all $\theta \in \mathcal{S}$ and for all $t>0$

$$
\left\{\begin{array}{l}
\psi(t, \theta)=\frac{1}{\phi(\theta)} c(t, \theta)^{-\sigma} \\
\psi_{t}(t, \theta)+D \psi_{x x}(t, \theta)+A(\theta)[\phi(\theta)-\nu(\theta)] \psi(t, \theta)-\rho \psi(t, \theta)+\mu(t, \theta)-\xi(t, \theta)=0 \\
\mu(t, \theta)\left[L(\theta)-L_{F}(t, \theta)\right]=0 \\
\xi(t, \theta) L_{F}(t, \theta)=0
\end{array}\right.
$$

Recalling that $\mathcal{L} \psi(t, \theta)=D \psi_{x x}(t, \theta)+A(\theta)[\phi(\theta)-\nu(\theta)] \psi(t, \theta)$, we can rewrite the second equation as

$$
\psi_{t}(t, \theta)+\mathcal{L} \psi(t, \theta)-\rho \psi(t, \theta)+\mu(t, \theta)-\xi(t, \theta)=0
$$

Note that if $L_{F}$ lies within its bounds, then both $\mu(t, \theta)$ and $\xi(t, \theta)$ are identically zero. Hence, we can provide a set of necessary conditions when fertile land is between the lower and the upper bound, 
at all locations

$$
I\left\{\begin{array}{l}
\frac{\partial L_{F}}{\partial t}(t, \theta)=\mathcal{L} L_{F}(t, \theta)-c(t, \theta) N(\theta) \phi(\theta), \\
\psi(t, \theta)=\frac{1}{\phi(\theta)} c(t, \theta)^{-\sigma} \\
\psi_{t}(t, \theta)+\mathcal{L} \psi(t, \theta)-\rho \psi(t, \theta)=0, \\
\lim _{t \rightarrow \infty} \psi(t, \theta) L_{F}(t, \theta)=0 .
\end{array}\right.
$$

Hence $(I)$ describes the optimal dynamics of the economy when no location has reached a bound.

The second phase is triggered whenever a location reaches the upper bound for fertile land. ${ }^{12}$ In this case, we assume that $\mathcal{S}=\underline{\mathcal{S}} \cup \overline{\mathcal{S}}$, where $\overline{\mathcal{S}}$ is the set of locations that have reached the upper bound. Hence, for each $\theta \in \overline{\mathcal{S}}$

$$
L_{F}(t, \theta)=L(\theta), \quad L_{F, t}(t, \theta)=0 \text { and } L_{F, x x}(t, \theta)=0,
$$

which implies that $c(t, \theta)=A(\theta) \frac{\phi(\theta)-\nu(\theta)}{\phi(\theta)} \frac{L(\theta)}{N(\theta)}, \forall t \geq 0$.

However, when analizing the set of optimal conditions in $\underline{S}$, we need to pay special attention to the border. The border condition (9) becomes

$$
\left.\psi(t, \theta) \nabla_{\theta} L_{F}(t, \theta)\right|_{\theta_{1}(t)} ^{\theta_{2}(t)}=\left.\nabla_{\theta} \psi(t, \theta) L_{F}(t, \theta)\right|_{\theta_{1}(t)} ^{\theta_{2}(t)} .
$$

Hence, in the second phase at all $t$

$$
I I\left\{\begin{array}{l}
\frac{\partial L_{F}}{\partial t}(t, \theta)=\mathcal{L} L_{F}(t, \theta)-c(t, \theta) N(\theta) \phi(\theta), \\
\psi(t, \theta)=\frac{1}{\phi(\theta)} c(t, \theta)^{-\sigma}, \\
\psi_{t}(t, \theta)+\mathcal{L} \psi(t, \theta)-\rho \psi(t, \theta)=0, \forall \theta \in \mathcal{S}, \\
\left.\psi(t, \theta) \nabla_{\theta} L_{F}(t, \theta)\right|_{\theta_{1}(t)} ^{\theta_{2}(t)}=\left.\nabla_{\theta} \psi(t, \theta) L_{F}(t, \theta)\right|_{\theta_{1}(t)} ^{\theta_{2}(t)}, \\
\lim _{t \rightarrow \infty} \psi(t, \theta) L_{F}(t, \theta)=0, \\
\text { and } \\
L_{F}(t, \theta)=L(\theta), \quad L_{F, t}(t, \theta)=0 \text { and } L_{F, x x}(t, \theta)=0, \\
c(t, \theta)=A(\theta) \frac{\phi(\theta)-\nu(\theta)}{\phi(\theta)} \frac{L(\theta)}{N(\theta)}, \forall \theta \in \overline{\mathcal{S}} .
\end{array}\right.
$$

\section{Proof of Proposition 1. Optimal solution in Phase 1.}

We prove that the explicit solution obtained using dynamic programming for the first phase fulfills the set of necessary conditions obtained via optimal control. This way, we are able to provide an explicit solution to the set of necessary conditions in (I). In Phase 1, the Hamilton-Jacobi-Bellman

\footnotetext{
${ }^{12}$ When fertile land reaches the lower bound, dynamics are similar to the case of the upper bound except that consumption becomes zero at locations with zero fertile land.
} 
(HJB) equation associated to the previous optimal control problem can be written as

$$
\rho v\left(L_{F}\right)=\left\langle L_{F}, \mathcal{L} \nabla v\left(L_{F}\right)\right\rangle+\sup _{c}\left\{\left\langle\frac{c^{1-\sigma}}{1-\sigma} N, \mathbb{1}\right\rangle-\left\langle\phi c N, \nabla v\left(L_{F}\right)\right\rangle\right\} .
$$

Let $\hat{c}:=\operatorname{argmax}\left\{\left\langle\frac{c^{1-\sigma}}{1-\sigma} N, \mathbb{1}\right\rangle-\left\langle\phi c N, \nabla v\left(L_{F}\right)\right\rangle\right\} . \hat{c}$ verifies that

$$
\hat{c}^{-\sigma} N=\phi N \nabla v\left(L_{F}\right) \Longleftrightarrow \hat{c}=\left[\phi \nabla v\left(L_{F}\right)\right]^{-\frac{1}{\sigma}} .
$$

Therefore

$$
\begin{aligned}
& \sup _{c}\left\{\left\langle\frac{c^{1-\sigma}}{1-\sigma} N, \mathbb{1}\right\rangle-\left\langle\phi c N, \nabla v\left(L_{F}\right)\right\rangle\right\}=\left\langle\frac{\hat{c}^{1-\sigma}}{1-\sigma} N, \mathbb{1}\right\rangle-\left\langle\phi \hat{c} N, \nabla v\left(L_{F}\right)\right\rangle \\
& =\left\langle\frac{1}{1-\sigma}\left[\phi \nabla v\left(L_{F}\right)\right]^{-\frac{1}{\sigma}} N, \mathbb{1}\right\rangle-\left\langle\phi N\left[\phi \nabla v\left(L_{F}\right)\right]^{-\frac{1}{\sigma}}, \nabla v\left(L_{F}\right)\right\rangle \\
& =\frac{1}{1-\sigma}\left\langle\left[\phi \nabla v\left(L_{F}\right)\right]^{-\frac{1-\sigma}{\sigma}} N, \mathbb{1}\right\rangle-\left\langle\left[\phi \nabla v\left(L_{F}\right)\right]^{-\frac{1-\sigma}{\sigma}} N, \mathbb{1}\right\rangle=\frac{\sigma}{1-\sigma}\left\langle\left[\phi \nabla v\left(L_{F}\right)\right]^{-\frac{1-\sigma}{\sigma}} N, \mathbb{1}\right\rangle .
\end{aligned}
$$

We look for a solution which can be written as

$$
v=\frac{\left\langle L_{F}, \alpha_{0} e_{0}\right\rangle^{1-\sigma}}{1-\sigma},
$$

with $\alpha_{0} \in \mathbb{R}$. Hence if $v$ is defined by (11), then $\nabla v\left(L_{F}\right)=\left\langle L_{F}, \alpha_{0} e_{0}\right\rangle^{-\sigma} \alpha_{0} e_{0}$. Plugging this solution in the HJB equation leads to the following equation in $\alpha_{0}$

$$
\begin{aligned}
& \frac{\rho}{1-\sigma}\left\langle L_{F}, \alpha_{0} e_{0}\right\rangle^{1-\sigma}=\left\langle L_{F},\left\langle L, \alpha_{0} e_{0}\right\rangle^{-\sigma} \mathcal{L} \alpha_{0} e_{0}\right\rangle+\frac{\sigma}{1-\sigma}\left\langle L_{F}, \alpha_{0} e_{0}\right\rangle^{1-\sigma}\left\langle\left(\phi \alpha_{0} e_{0}\right)^{-\frac{1-\sigma}{\sigma}} N, \mathbb{1}\right\rangle \\
& =\lambda_{0}\left\langle L_{F}, \alpha_{0} e_{0}\right\rangle^{1-\sigma}+\frac{\sigma}{1-\sigma}\left\langle L_{F}, \alpha_{0} e_{0}\right\rangle^{1-\sigma} \int_{0}^{2 \pi}\left[\phi(\eta) \alpha_{0} e_{0}(\eta)\right]^{-\frac{1-\sigma}{\sigma}} N(\eta) d \eta .
\end{aligned}
$$

Dividing by $\left\langle L_{F}, \alpha_{0} e_{0}\right\rangle^{1-\sigma}$

$$
\begin{gathered}
\frac{\rho}{1-\sigma}=\lambda_{0}+\frac{\sigma}{1-\sigma} \int_{0}^{2 \pi}\left[\phi(\eta) \alpha_{0} e_{0}(\eta)\right]^{-\frac{1-\sigma}{\sigma}} N(\eta) d \eta \\
\Longleftrightarrow \frac{\rho-\lambda_{0}(1-\sigma)}{\sigma}=\alpha_{0}^{-\frac{1-\sigma}{\sigma}} \int_{0}^{2 \pi}\left[\phi(\eta) e_{0}(\eta)\right]^{-\frac{1-\sigma}{\sigma}} N(\eta) d \eta .
\end{gathered}
$$

Since by Assumption $2 \rho-\lambda_{0}(1-\sigma)>0$, then $\alpha_{0}$ obtains as

$$
\alpha_{0}=\left[\frac{\sigma}{\rho-\lambda_{0}(1-\sigma)} \int_{0}^{2 \pi}\left[\phi(\eta) e_{0}(\eta)\right]^{-\frac{1-\sigma}{\sigma}} N(\eta) d \eta\right]^{\frac{\sigma}{1-\sigma}} .
$$

Moreover, we have that

$$
L_{F}^{\prime}=\mathcal{L} L_{F}-(\phi N)^{\frac{\sigma-1}{\sigma}} N^{\frac{1}{\sigma}}\left(\alpha_{0} e_{0}\right)^{-\frac{1}{\sigma}}\left\langle L_{F}, \alpha_{0} e_{0}\right\rangle
$$


Thus $\forall \Phi \in \mathcal{D}(\mathcal{L})$

$$
\begin{aligned}
\frac{d}{d t}\left\langle L_{F}, \Phi\right\rangle & =\left\langle\mathcal{L} L_{F}, \Phi\right\rangle-\left\langle L, \alpha_{0} e_{0}\right\rangle\left\langle(\phi N)^{\frac{\sigma-1}{\sigma}} N^{\frac{1}{\sigma}}\left(\alpha_{0} e_{0}\right)^{-\frac{1}{\sigma}}, \Phi\right\rangle \\
& =\left\langle L_{F}, \mathcal{L} \Phi\right\rangle-\left\langle L, \alpha_{0} e_{0}\right\rangle\left\langle(\phi N)^{\frac{\sigma-1}{\sigma}} N^{\frac{1}{\sigma}}\left(\alpha_{0} e_{0}\right)^{-\frac{1}{\sigma}}, \Phi\right\rangle
\end{aligned}
$$

In particular, for $\Phi=\alpha_{0} e_{0}$

$$
\begin{aligned}
\frac{d}{d t}\left\langle L_{F}, \alpha_{0} e_{0}\right\rangle & =\left\langle L_{F}, \lambda_{0} \alpha_{0} e_{0}\right\rangle-\left\langle L_{F}, \alpha_{0} e_{0}\right\rangle\left\langle(\phi N)^{\frac{\sigma-1}{\sigma}} N^{\frac{1}{\sigma}}\left(\alpha_{0} e_{0}\right)^{-\frac{1}{\sigma}}, \alpha_{0} e_{0}\right\rangle \\
& =\left[\lambda_{0}-\left\langle(\phi N)^{\frac{\sigma-1}{\sigma}} N^{\frac{1}{\sigma}}\left(\alpha_{0} e_{0}\right)^{-\frac{1}{\sigma}}, \alpha_{0} e_{0}\right\rangle\right]\left\langle L_{F}, \alpha_{0} e_{0}\right\rangle .
\end{aligned}
$$

Let $g$ be $g:=\lambda_{0}-\left\langle(\phi N)^{\frac{\sigma-1}{\sigma}} N^{\frac{1}{\sigma}}\left(\alpha_{0} e_{0}\right)^{-\frac{1}{\sigma}}, \alpha_{0} e_{0}\right\rangle$. Then we can write the expression above as

$$
\frac{d}{d t}\left\langle L_{F}, \alpha_{0} e_{0}\right\rangle=g\left\langle L_{F}, \alpha_{0} e_{0}\right\rangle
$$

whose solution is $\left\langle L_{F}, \alpha_{0} e_{0}\right\rangle=\left\langle L_{F}^{0}, \alpha_{0} e_{0}\right\rangle e^{g t}$, where $L_{F}^{0}$ is the initial distribution for fertile soil. Optimal consumption can be expressed as

$$
\left.c^{*}=\left[\phi \nabla v\left(L_{F}\right)\right]^{-\frac{1}{\sigma}}=\phi^{-\frac{1}{\sigma}}\left[\left\langle L_{F}, \alpha_{0} e_{0}\right\rangle^{-\sigma} \alpha_{0} e_{0}\right\rangle\right]^{-\frac{1}{\sigma}}=\phi^{-\frac{1}{\sigma}}\left\langle L_{F}, \alpha_{0} e_{0}\right\rangle\left(\alpha_{0} e_{0}\right)^{-\frac{1}{\sigma}} .
$$

Using (26) we obtain optimal consumption in terms of the initial distribution of fertile land, the model parameters and $g$

$$
c^{*}=\phi^{-\frac{1}{\sigma}}\left(\alpha_{0} e_{0}\right)^{-\frac{1}{\sigma}}\left\langle L_{F}^{0}, \alpha_{0} e_{0}\right\rangle e^{g t} .
$$

Finally when we obtained the expression of $\alpha_{0}$, it was shown that

$$
\frac{\rho-\lambda_{0}(1-\sigma)}{\sigma}=\int_{0}^{2 \pi}\left[\phi(z) N(z) \alpha_{0} e_{0}(z)\right]^{-\frac{1-\sigma}{\sigma}} N(z)^{\frac{1}{\sigma}} d z .
$$

Therefore, a simpler expression of $g$ can be derived

$$
g=\lambda_{0}-\int_{0}^{2 \pi}\left[\phi(z) N(z) \alpha_{0} e_{0}(z)\right]^{-\frac{1-\sigma}{\sigma}} N(z)^{\frac{1}{\sigma}} d z=\lambda_{0}-\frac{\rho-\lambda_{0}(1-\sigma)}{\sigma}=\frac{\lambda_{0}-\rho}{\sigma} .
$$

With this explicit solution in hand, the next step is to prove that it actually verifies the set of necessary conditions in $(I)$. We claim that:

$$
c^{*}(t, \theta)=\left[\phi(\theta) \alpha_{0} e_{0}(\theta)\right]^{-\frac{1}{\sigma}} e^{g t} \int_{0}^{2 \pi} L_{F}^{0}(z) \alpha_{0} e_{0}(z) d z,
$$

is the optimal solution in Phase 1. As said, let us prove next that actually this solution verifies the optimal necessary conditions $(I)$. Since $\psi(t, \theta)=\frac{1}{\phi(\theta)} c(t, \theta)^{-\sigma}$, then

$$
\psi(t, \theta)=\alpha_{0} e_{0}(\theta) e^{-\sigma g t}\left(\int_{0}^{2 \pi} L_{F}^{0}(z) \alpha_{0} e_{0}(z) d z\right)^{-\sigma}
$$


and its partial derivatives are

$$
\begin{aligned}
& \psi_{t}(t, \theta)=-\sigma g \psi(t, \theta), \\
& \psi_{x}(t, \theta)=\alpha_{0} e_{0}^{\prime}(\theta) e^{-\sigma g t}\left(\int_{0}^{2 \pi} L_{F}^{0}(z) \alpha_{0} e_{0}(z) d z\right)^{-\sigma}, \\
& \psi_{x x}(t, \theta)=\alpha_{0} e_{0}^{\prime \prime}(\theta) e^{-\sigma g t}\left(\int_{0}^{2 \pi} L_{F}^{0}(z) \alpha_{0} e_{0}(z) d z\right)^{-\sigma} .
\end{aligned}
$$

Substituting into

$$
\psi_{t}(t, \theta)+\mathcal{L} \psi(t, \theta)-\rho \psi(t, \theta)+\mu(t, \theta)=0,
$$

checking that $\mathcal{L} \psi(t, \theta)=\lambda_{0} \psi(t, \theta)$, and letting $\mu(t, \theta) \equiv 0$, we do obtain that the solution in (30) satisfies the optimality condition whenever $g=\frac{\lambda_{0}-\rho}{\sigma}$.

Finally, once we have proven that both solutions coincide, we close this proof showing that aggregated land grows at rate $g$. Since $\left\langle L_{F}, \alpha_{0} e_{0}\right\rangle=\left\langle L_{F}^{0}, \alpha_{0} e_{0}\right\rangle e^{g t}$ then

$$
\int_{0}^{2 \pi} L_{F}(t, \theta) e_{0}(\theta) d \theta=\int_{0}^{2 \pi} L_{F}^{0}(\theta) e_{0}(\theta) e^{g t} d \theta \Longleftrightarrow \int_{0}^{2 \pi}\left[L_{F}(t, \theta)-L_{F}^{0}(\theta) e^{g t}\right] e_{0}(\theta) d \theta=0 .
$$

Defining $\underline{e_{0}}:=\min _{[0,2 \pi]} e_{0}(\theta)$ and $\overline{e_{0}}:=\max _{[0,2 \pi]} e_{0}(\theta)$, the following inequalities obtain

$$
\underline{e_{0}} \int_{0}^{2 \pi}\left[L_{F}(t, \theta)-L_{F}^{0}(\theta) e^{g t}\right] d \theta \leq \int_{0}^{2 \pi}\left[L_{F}(t, \theta)-L_{F}^{0}(\theta) e^{g t}\right] e_{0}(\theta) d \theta=0,
$$

and

$$
\overline{e_{0}} \int_{0}^{2 \pi}\left[L_{F}(t, \theta)-L_{F}^{0}(\theta) e^{g t}\right] d \theta \geq \int_{0}^{2 \pi}\left[L_{F}(t, \theta)-L_{F}^{0}(\theta) e^{g t}\right] e_{0}(\theta) d \theta=0 .
$$

Hence, since $e_{0}(\theta)>0 \forall \theta \in[0,2 \pi]$, then (13) and (14) imply that $\overline{e_{0}}>0$ and $\underline{e_{0}}>0$. As a result, (13) and (14) imply that

$$
\int_{0}^{2 \pi}\left[L_{F}(t, \theta)-L_{F}^{0}(\theta) e^{g t}\right] d \theta \leq 0, \text { and } \int_{0}^{2 \pi}\left[L_{F}(t, \theta)-L_{F}^{0}(\theta) e^{g t}\right] d \theta \geq 0,
$$

which implies that $\int_{0}^{2 \pi}\left(L_{F}(t, \theta)-L_{F}^{0}(\theta) e^{g t}\right) d \theta=0$. Dividing by the length of the interval, the result in terms of mean values obtains: $\left\langle L_{F}(t)\right\rangle=e^{g t}\left\langle L_{F}^{0}\right\rangle$.

\section{Proof of Proposition 2.}

We decompose fertile soil on the eigenvector basis as $L_{F}=\sum_{n \geq 0}\left\langle L_{F}, e_{n}\right\rangle e_{n}(\theta)$ and look for the expression of the coefficients $\left\langle L_{F}, e_{n}\right\rangle$ for $n \geq 0$. Recall that $A, \phi$ and $\nu, N \in \mathbb{R}$. Thus, the eigenvalue problem can be written as the following second order linear ordinary differential equation with constant coefficients

$$
u^{\prime \prime}+\frac{A(\phi-\nu)-\lambda}{D} u=0 .
$$

Any function $u(\theta)=C_{1} \cos \left(\theta \sqrt{\frac{A(\phi-\nu)-\lambda}{D}}+C_{2}\right)$, whith $\left(C_{1}, C_{2}\right) \in \mathbb{R}^{2}$ is a solution to (15). Note that since our problem is $2 \pi$ periodic, solutions obviously need to verify $u(0)=u(2 \pi)$. Imposing this 
boundary condition, we obtain that

$$
\cos \left(C_{2}\right)=\cos \left(2 \pi \sqrt{\frac{A(\phi-\nu)-\lambda}{D}}+C_{2}\right),
$$

which holds if and only if

$$
2 \pi \sqrt{\frac{A(\phi-\nu)-\lambda_{n}}{D}}+C_{2}=C_{2}+2 n \pi, \text { with } n \in \mathbb{Z} \Longrightarrow \sqrt{\frac{A(\phi-\nu)-\lambda_{n}}{D}}=n .
$$

Therefore, the $n^{\text {th }}$ eigenvalue of the problem and the associated $n^{\text {th }}$ eigenvector are $\lambda_{n}=A(\phi-\nu)-$ $D n^{2}$, and

$$
\begin{aligned}
e_{n}(\theta) & =C_{1} \cos \left(\theta \sqrt{\frac{A(\phi-\nu)-\lambda_{n}}{D}}+C_{2}\right)=C_{1} \cos \left(\theta \sqrt{\frac{A(\phi-\nu)-A(\phi-\nu)+D n^{2}}{D}}+C_{2}\right) \\
& =C_{1} \cos \left(\theta n+C_{2}\right) .
\end{aligned}
$$

Here we need to analyze separatedly the cases $n=0$ and $n>0$ :

1. When $n=0$, we have that $\lambda_{0}=A(\phi-\nu)$. As a result, $g=\frac{\lambda_{0}-\rho}{\sigma}=\frac{A(\phi-\nu)-\rho}{\sigma}$. From (16), $e_{0}(\theta)=e_{0}=C_{1} \cos \left(C_{2}\right)$ is spatially homogeneous. As a result, $\left\langle L_{F}, e_{0}\right\rangle e_{0}$ can be written as

$$
\left\langle L_{F}, e_{0}\right\rangle e_{0}=\left\langle L_{F}^{0}, e_{0}\right\rangle e_{0} e^{g t}=e_{0}^{2} \int_{0}^{2 \pi} L_{F}^{0}(\theta) d \theta e^{g t} .
$$

Since eigenvectors form an orthonormal basis, we have that in particular $\int_{0}^{2 \pi} e_{0}^{2} d \theta=1$, which implies that $e_{0}^{2}=\frac{1}{2 \pi}$. As a result, we can write $\left\langle L_{F}, e_{0}\right\rangle e_{0}=\left\langle L_{F}^{0}, e_{0}\right\rangle e_{0} e^{g t}=\left\langle L_{F}^{0}\right\rangle e^{g t}$.

2. For $n \geq 1$, the dynamics of fertile soil is given by

$$
L_{F}^{\prime}=\mathcal{L} L_{F}-(\phi N)^{\frac{\sigma-1}{\sigma}} N^{\frac{1}{\sigma}}\left(\alpha_{0} e_{0}\right)^{-\frac{1}{\sigma}}\left\langle L_{F}, \alpha_{0} e_{0}\right\rangle,
$$

and $\left\langle L_{F}, e_{0}\right\rangle=\left\langle L_{F}^{0}, e_{0}\right\rangle e^{g t}$, so that

$$
\begin{aligned}
\frac{d}{d t}\left\langle L_{F}, e_{n}\right\rangle & =\left\langle\mathcal{L} L_{F}, e_{n}\right\rangle-\left\langle L_{F}, \alpha_{0} e_{0}\right\rangle\left\langle(\phi N)^{\frac{\sigma-1}{\sigma}} N^{\frac{1}{\sigma}}\left(\alpha_{0} e_{0}\right)^{-\frac{1}{\sigma}}, e_{n}\right\rangle \\
& =\left\langle L_{F}, \mathcal{L} e_{n}\right\rangle-\left\langle L_{F}, \alpha_{0} e_{0}\right\rangle \beta_{n}=\lambda_{n}\left\langle L_{F}, e_{n}\right\rangle-\left\langle L_{F}^{0}, \alpha_{0} e_{0}\right\rangle e^{g t} \beta_{n}
\end{aligned}
$$

with $\beta_{n}=\left\langle(\phi N)^{\frac{\sigma-1}{\sigma}} N^{\frac{1}{\sigma}}\left(\alpha_{0} e_{0}\right)^{-\frac{1}{\sigma}}, e_{n}\right\rangle$. Since $\phi, N$, and $e_{0}$ are spatially homogeneous

$$
\begin{aligned}
\beta_{n} & =(\phi N)^{\frac{\sigma-1}{\sigma}} N^{\frac{1}{\sigma}}\left(\alpha_{0} e_{0}\right)^{-\frac{1}{\sigma}} \int_{0}^{2 \pi} e_{n}(\theta) d \theta=(\phi N)^{\frac{\sigma-1}{\sigma}} N^{\frac{1}{\sigma}}\left(\alpha_{0} e_{0}\right)^{-\frac{1}{\sigma}} \int_{0}^{2 \pi} C_{1} \cos \left(\theta n+C_{2}\right) d \theta \\
& =(\phi N)^{\frac{\sigma-1}{\sigma}} N^{\frac{1}{\sigma}}\left(\alpha_{0} e_{0}\right)^{-\frac{1}{\sigma}}\left[\frac{C_{1}}{n} \sin \left(\theta n+C_{2}\right)\right]_{0}^{2 \pi}=0 .
\end{aligned}
$$

Therefore, $\frac{d}{d t}\left\langle L_{F}, e_{n}\right\rangle=\lambda_{n}\left\langle L_{F}, e_{n}\right\rangle$, and $\left\langle L_{F}, e_{n}\right\rangle=\left\langle L_{F}^{0}, e_{n}\right\rangle e^{\lambda_{n} t}$.

Substituting $\left\langle L_{F}, e_{0}\right\rangle e_{0}$ and $\left\langle L_{F}, e_{n}\right\rangle e_{n}$ into $L_{F}=\sum_{n \geq 0}\left\langle L_{F}, e_{n}\right\rangle e_{n}(\theta), L_{F}$ can be written as $L_{F}(t, \theta)=\left\langle L_{F}^{0}\right\rangle e^{g t}+\sum_{n \geq 1}\left\langle L_{F}^{0}, e_{n}\right\rangle e_{n} e^{\lambda_{n} t}$, which proves our claim. 


\section{E. Proof of Proposition 3. Consumption transfers.}

This proof is structured in three steps. First, we obtain the optimal solution for the policy maker problem in the un-connected economy. Second, we do the same for the diffusive economy. In the third step, we use the previous results to compute consumption transfers.

1. We obtain optimal consumption $c_{U}(t)$ in the unconnected economy. Here, the social planner solves the following standard Ramsey-type problem for each location

$$
\max _{c_{U}(t)} \int_{0}^{\infty} \frac{c_{U}^{1-\sigma}(t, \theta)}{1-\sigma} N e^{-\rho t} d t
$$

subject to

$$
\left\{\begin{array}{l}
\dot{L}_{F}(t, \theta)=A(\phi-\nu) L_{F}(t, \theta)-\Phi N c_{U}(t, \theta) \\
L_{F}(0, \theta)=L_{F}^{0}(\theta) \geq 0
\end{array}\right.
$$

for all $t>0$ and $\theta \in \mathcal{S}$, with $N, A, \phi, \nu, \Phi \in \mathbb{R}$. The Hamiltonian associated to (17)-(18) writes as $\mathcal{H}\left(c_{U}, L_{F}, \lambda, t\right)=\frac{c_{U}^{1-\sigma}}{1-\sigma} N e^{-\rho t}+\zeta\left[A(\phi-\nu) L_{F}-\Phi N c_{U}\right]$. Taking the first order conditions of $\mathcal{H}$ with respect to $c_{U}$ and $L_{F}$, we obtain the set of Pontryagin conditions

$$
\left\{\begin{array}{l}
\frac{\partial \mathcal{H}}{\partial c_{U}}=0 \Longrightarrow c_{U}^{-\sigma} N e^{-\rho t}=\zeta \phi N \\
\frac{\partial \mathcal{H}}{\partial L_{F}}=-\dot{\zeta} \Longrightarrow-\dot{\lambda}=\zeta A(\phi-\nu)
\end{array}\right.
$$

plus the transversality condition $\lim _{t \rightarrow \infty} L_{F}(t, \theta) \zeta(t)=0$. Let us define $\tilde{\zeta}=\zeta e^{\rho t}$. Then we can express the dynamics of the co-state variable as $\dot{\tilde{\zeta}}=-\tilde{\zeta}(A(\phi-\nu)-\rho)$. Since $\tilde{\zeta}=\frac{c_{U}^{-\sigma}}{\phi}$, then $\dot{\tilde{\zeta}}=-\frac{\sigma c_{U}^{-\sigma-1} \dot{c}_{U}}{\phi}$ and $\frac{\dot{\tilde{\zeta}}}{\widetilde{\zeta}}=-\sigma \frac{\dot{c}_{U}}{c_{U}}$.

$g_{U}$ denotes $c_{U}^{\prime} s$ growth rate, $g_{U}:=\frac{\dot{c}_{U}}{c_{U}}=\frac{A(\phi-\nu)-\rho}{\sigma}$ and $c_{U}(t, \theta)=c_{U}(0, \theta) e^{g t}$. Note that $g_{U}=g$, which proves that location's consumption in the unconnected and the diffusive economy grow at the same rate. Hereafter we will write $g$.

Finally, note that

$$
\dot{L}_{F}(t, \theta)=A(\phi-\nu) L_{F}(t, \theta)-\phi N c_{U}(t, \theta) \Longleftrightarrow \frac{\dot{L}_{F}(t, \theta)}{L_{F}(t, \theta)}=A(\phi-\nu)-\phi N \frac{c_{U}(t, \theta)}{L_{F}(t, \theta)} .
$$

Hence $\frac{\dot{L}_{F}(t, \theta)}{L_{F}(t, \theta)}=g$ along the balanced growth path, and as a result $L_{F}(t, \theta)=L_{F}(0, \theta) e^{g t}=L_{F}^{0}(\theta) e^{g t}$, and

$$
c_{U}(t, \theta)=\frac{A}{N \sigma \phi}\left[\frac{\rho}{A}+(\phi-\nu)(\sigma-1)\right] L_{F}(t, \theta)=\frac{A}{N \sigma \phi}\left[\frac{\rho}{A}+(\phi-\nu)(\sigma-1)\right] L_{F}^{0}(\theta) e^{g t} .
$$

2. Proposition 1 provided us with the trajectory for optimal consumption in the diffusive economy, $c_{D}$

$$
c_{D}(t, \theta)=e^{g t}\left(\phi \alpha_{0} e_{0}(\theta)\right)^{-\frac{1}{\sigma}} \int_{0}^{2 \pi} L_{F}^{0}(\eta) \alpha_{0} e_{0}(\eta) d \eta
$$

with

$$
\alpha_{0}=\left[\frac{\sigma}{\rho-A(\phi-\nu)(1-\sigma)} \int_{0}^{2 \pi} N\left(\phi e_{0}(\eta)\right)^{-\frac{1-\sigma}{\sigma}} d \eta\right]^{\frac{\sigma}{1-\sigma}}
$$


and where $e_{0}(\cdot)$ the first eigenvector of the problem $\mathcal{L} u=\lambda u$. In Appendix $\mathrm{D}$ we proved that $e_{0}$ is spatially homogeneous, so that

$$
\begin{aligned}
\alpha_{0} e_{0} & =\left[\frac{\sigma}{\rho-A(\phi-\nu)(1-\sigma)} \int_{0}^{2 \pi} N\left(\phi e_{0}\right)^{-\frac{1-\sigma}{\sigma}} d \eta\right]^{\frac{\sigma}{1-\sigma}} e_{0} \\
& =\left[\frac{2 \pi \sigma N\left(\phi e_{0}\right)^{-\frac{1-\sigma}{\sigma}}}{\rho-A(\phi-\nu)(1-\sigma)}\right]^{\frac{\sigma}{1-\sigma}} e_{0}=\frac{1}{\phi}\left[\frac{2 \pi \sigma N}{\rho-A(\phi-\nu)(1-\sigma)}\right]^{\frac{\sigma}{1-\sigma}} .
\end{aligned}
$$

Therefore, we can prove that $c_{D}$ is homogeneous in space and we obtain the following expression

$$
\begin{aligned}
c_{D}(t, \theta) & =e^{g t}\left(\phi \alpha_{0} e_{0}\right)^{-\frac{1}{\sigma}} \int_{0}^{2 \pi} L_{F}^{0}(\eta) \alpha_{0} e_{0} d \eta \\
& =e^{g t}\left[\frac{2 \pi \sigma N}{\rho-A(\phi-\nu)(1-\sigma)}\right]^{\frac{1}{\sigma-1}} \frac{1}{\phi}\left[\frac{2 \pi \sigma N}{\rho-A(\phi-\nu)(1-\sigma)}\right]^{\frac{\sigma}{1-\sigma}} \int_{0}^{2 \pi} L_{F}^{0}(\eta) d \eta \\
& =e^{g t} \frac{\rho-A(\phi-\nu)(1-\sigma)}{2 \pi \sigma N \phi} \int_{0}^{2 \pi} L_{F}^{0}(\eta) d \eta=\frac{\rho-A(\phi-\nu)(1-\sigma)}{N \sigma \phi}\left\langle L_{F}^{0}\right\rangle e^{g t} .
\end{aligned}
$$

3. Finally, let us compute consumption transfers. $\Gamma(t, \theta)$ obtains using all previous results

$$
\begin{aligned}
\Gamma(t, \theta) & =c_{U}(t, \theta)-c_{D}(t, \theta)=\frac{\rho+A(\phi-\nu)(\sigma-1)}{N \sigma \phi} L_{F}^{0}(\theta) e^{g t}-\frac{\rho+A(\phi-\nu)(\sigma-1)}{N \sigma \phi}\left\langle L_{F}^{0}\right\rangle e^{g t} \\
& =\frac{\rho+A(\phi-\nu)(\sigma-1)}{N \sigma \phi}\left[L_{F}^{0}(\theta)-\left\langle L_{F}^{0}\right\rangle\right] e^{g t} .
\end{aligned}
$$

\section{F. Proof of Proposition 4. Optimal solution in Phase 2.}

In order to obtain the optimal trajectories in $\underline{S}(t)$, we need to start by defining the interior product in $\underline{S}(t)$. For any two functions $f, g$ in $\mathcal{H}^{2}(\underline{\mathcal{S}}(t))$, we define their interior product as

$$
\langle f, g\rangle_{\underline{S}}=\int_{\theta_{1}(t)}^{\theta_{2}(t)} f(z) g(z) d z .
$$

In Phase 2, the HJB equation is

$$
\rho v\left(L_{F}\right)=\left\langle L_{F}, \mathcal{L} \nabla v\left(L_{F}\right)\right\rangle_{\underline{S}}+\sup _{c}\left\{\left\langle{\frac{c^{1-\sigma}}{1-\sigma}}^{N}, \mathbb{1}\right\rangle_{\underline{S}}-\left\langle\phi c N, \nabla v\left(L_{F}\right)\right\rangle_{\underline{S}}\right\} .
$$

Let $\hat{c}:=\operatorname{argmax}\left\{\left\langle\frac{c^{1-\sigma}}{1-\sigma} N, \mathbb{1}\right\rangle_{\underline{S}}-\left\langle\phi c N, \nabla v\left(L_{F}\right)\right\rangle_{\underline{S}}\right\}$. As in Appendix C, $\hat{c}$ verifies that $\hat{c}=\left[\phi \nabla v\left(L_{F}\right)\right]^{-\frac{1}{\sigma}}$.

As in Phase 1, we also look in Phase 2 for a solution that can be written as $v=\frac{\left\langle L_{F}, \beta_{0} e_{0}\right\rangle_{\underline{S}}^{1-\sigma}}{1-\sigma}$, where $\beta_{0}$ is this time a continuous function of time. $e_{0}$ is the first eigenvector associated to eigenvalue $\lambda_{0}$ of the eigenvalue problem: $\mathcal{L} u=\lambda u$ on $S$. Note that we are borrowing the first eigenvector and eigenvalue from the problem on $S$ and not restricting the eigenvalue problem to $\underline{S}$. 
Operator $\mathcal{L}$ is self-adjoint on $\underline{S}$ only under certain assumptions. Indeed

$$
\begin{aligned}
\left\langle\mathcal{L} L_{F}, \nabla v\left(L_{F}\right)\right\rangle_{\underline{S}} & =\int_{\theta_{1}(t)}^{\theta_{2}(t)} \mathcal{L} L_{F}(z) \nabla v\left(L_{F}\right)(z) d z \\
& =\left.\nabla_{\theta} L_{F}(z) \nabla v\left(L_{F}\right)\right|_{\theta_{1}(t)} ^{\theta_{2}(t)}-\int_{\theta_{1}(t)}^{\theta_{2}(t)} \nabla_{\theta} L_{F}(z) \nabla_{\theta}\left(\nabla v\left(L_{F}\right)(z)\right) d z \\
& =\left.\nabla_{\theta} L_{F}(z) \nabla v\left(L_{F}\right)\right|_{\theta_{1}(t)} ^{\theta_{2}(t)}-\left.L_{F}(z) \nabla_{\theta}\left(\nabla v\left(L_{F}\right)\right)\right|_{\theta_{1}(t)} ^{\theta_{2}(t)}+\int_{\theta_{1}(t)}^{\theta_{2}(t)} L_{F}(z) \mathcal{L} \nabla v\left(L_{F}\right)(z) d z .
\end{aligned}
$$

Hence

$$
\left\langle\mathcal{L} L_{F}, \nabla v\left(L_{F}\right)\right\rangle_{\underline{S}}=\left\langle L_{F}, \mathcal{L}\left(\nabla v\left(L_{F}\right)\right)\right\rangle_{\underline{S}}+\left.\nabla_{\theta} L_{F}(z) \nabla v\left(L_{F}\right)\right|_{\theta_{1}(t)} ^{\theta_{2}(t)}-\left.L_{F}(z) \nabla_{\theta}\left(\nabla v\left(L_{F}\right)\right)\right|_{\theta_{1}(t)} ^{\theta_{2}(t)},
$$

and our operator is self-adjoint if and only if

$$
\left.\nabla_{\theta} L_{F}(z) \nabla v\left(L_{F}\right)\right|_{\theta_{1}(t)} ^{\theta_{2}(t)}=\left.L_{F}(z) \nabla_{\theta}\left(\nabla v\left(L_{F}\right)\right)\right|_{\theta_{1}(t)} ^{\theta_{2}(t)} .
$$

Note that (19) coincides with (10), implying that operator $\mathcal{L}$ is self-adjoint in the subset of optimal solutions.

Proceeding like in Appendix C, we substitute $\hat{c}$ into the HJB equation and impose that the solutions must be optimal, that is, that they verify (19):

$$
\begin{aligned}
& \frac{\rho}{1-\sigma}\left\langle L_{F}, \beta_{0} e_{0}\right\rangle_{\underline{S}}^{1-\sigma}=\left\langle L_{F},\left\langle L, \beta_{0} e_{0}\right\rangle^{-\sigma} \mathcal{L} \beta_{0} e_{0}\right\rangle_{\underline{S}}+\frac{\sigma}{1-\sigma}\left\langle L_{F}, \beta_{0} e_{0}\right\rangle_{\underline{S}}^{1-\sigma}\left\langle\left(\phi \beta_{0} e_{0}\right)^{-\frac{1-\sigma}{\sigma}} N, \mathbb{1}\right\rangle_{\underline{S}} \\
& =\lambda_{0}\left\langle L_{F}, \beta_{0} e_{0}\right\rangle^{1-\sigma}+\frac{\sigma}{1-\sigma}\left\langle L_{F}, \beta_{0} e_{0}\right\rangle_{\underline{S}}^{1-\sigma} \int_{\underline{S}}\left(\phi(\eta) \beta_{0} e_{0}(\eta)\right)^{-\frac{1-\sigma}{\sigma}} N(\eta) d \eta .
\end{aligned}
$$

Hence, dividing on both sides by $\left\langle L_{F}, \beta_{0} e_{0}\right\rangle_{\underline{S}}^{1-\sigma}$ :

$$
\begin{gathered}
\frac{\rho}{1-\sigma}=\lambda_{0}+\frac{\sigma}{1-\sigma} \int_{\underline{S}}\left(\phi(\eta) \beta_{0} e_{0}(\eta)\right)^{-\frac{1-\sigma}{\sigma}} N(\eta) d \eta \\
\Longleftrightarrow \frac{\rho-\lambda_{0}(1-\sigma)}{\sigma}=\beta_{0}^{-\frac{1-\sigma}{\sigma}} \int_{\underline{S}}\left(\phi(\eta) e_{0}(\eta)\right)^{-\frac{1-\sigma}{\sigma}} N(\eta) d \eta .
\end{gathered}
$$

By Assumption 2, $\rho-\lambda_{0}(1-\sigma)>0$, so that

$$
\beta_{0}^{\frac{1-\sigma}{\sigma}}=\frac{\sigma}{\rho-\lambda_{0}(1-\sigma)} \int_{\underline{S}}\left(\phi(z) e_{0}(z)\right)^{-\frac{1-\sigma}{\sigma}} N(z) d z=\frac{\sigma}{\rho-\lambda_{0}(1-\sigma)}<\left(\phi(z) e_{0}(z)\right)^{-\frac{1-\sigma}{\sigma}} N(z), \mathbb{1}>_{\underline{S}} .
$$

As a result, we can write $\hat{c}$ as $\hat{c}=\left[\phi \nabla v\left(L_{F}\right)\right]^{-\frac{1}{\sigma}}=\left(\phi \beta_{0} e_{0}\right)^{-\frac{1}{\sigma}}<L_{F}, \beta_{0} e_{0}>_{\underline{S}}$.

Taking the logarithm of (20) and derivating, we obtain $\beta_{0}$ 's growth rate

$$
\begin{aligned}
\frac{1-\sigma}{\sigma} \frac{\beta_{0, t}(t)}{\beta_{0}(t)} & =\frac{d}{d t} \ln \int_{\underline{S}}\left(\phi(z) e_{0}(z)\right)^{-\frac{1-\sigma}{\sigma}} N(z) d z \\
& =\frac{N\left(\theta_{2}(t)\right)\left[\phi\left(\theta_{2}(t)\right) e_{0}\left(\theta_{2}(t)\right)\right]^{-\frac{1-\sigma}{\sigma}} \dot{\theta}_{2}(t)-N\left(\theta_{1}(t)\right)\left[\phi\left(\theta_{1}(t)\right) e_{0}\left(\theta_{1}(t)\right)\right]^{-\frac{1-\sigma}{\sigma}} \dot{\theta}_{1}(t)}{\int_{\underline{S}}\left(\phi(z) e_{0}(z)\right)^{-\frac{1-\sigma}{\sigma}} N(z) d z} .
\end{aligned}
$$


Replacing $\hat{c}$ in the original state equation for $L_{F}$, we obtain that

$$
L_{F}^{\prime}=\mathcal{L} L_{F}-\phi N\left(\phi \beta_{0} e_{0}\right)^{-\frac{1}{\sigma}}\left\langle L_{F}, \beta_{0} e_{0}\right\rangle_{\underline{S}} .
$$

Let us multiply (22) by $\beta_{0} e_{0}$ and compute the interior product restricting our analysis to optimal solutions that verify (19)

$$
\begin{aligned}
\left\langle\frac{d}{d t} L_{F}, \beta_{0} e_{0}\right\rangle_{\underline{S}} & =\left\langle\mathcal{L} L_{F}, \beta_{0} e_{0}\right\rangle_{\underline{S}}-\left\langle(\phi N)^{\frac{\sigma-1}{\sigma}} N^{\frac{1}{\sigma}}\left(\beta_{0} e_{0}\right)^{-\frac{1}{\sigma}}, \beta_{0} e_{0}\right\rangle_{\underline{S}}\left\langle L_{F}, \beta_{0} e_{0}\right\rangle_{\underline{S}} \\
& =\left\langle L_{F}, \beta_{0} \mathcal{L} e_{0}\right\rangle_{\underline{S}}-\left\langle\left(\phi N \beta_{0} e_{0}\right)^{\frac{-(1-\sigma)}{\sigma}} N^{\frac{1}{\sigma}}, \underline{1}\right\rangle_{\underline{S}}\left\langle L_{F}, \beta_{0} e_{0}\right\rangle_{\underline{S}} \\
& =\lambda_{0}\left\langle L_{F}, \beta_{0} e_{0}\right\rangle_{\underline{S}}-\left\langle\left(\phi N \beta_{0} e_{0}\right)^{\frac{-(1-\sigma)}{\sigma}} N^{\frac{1}{\sigma}}, \mathbb{1}\right\rangle_{\underline{S}}\left\langle L_{F}, \beta_{0} e_{0}\right\rangle_{\underline{S}} .
\end{aligned}
$$

Note that we cannot solve the problem unless we transform $\left\langle\frac{d}{d t} L_{F}, \beta_{0} e_{0}\right\rangle_{\underline{S}}$. Indeed, when the interior product changes with time, it is not straightforward to proceed as in Appendix C. Note that

$$
\begin{aligned}
\frac{d}{d t}\left\langle L_{F}, \beta_{0} e_{0}\right\rangle_{\underline{S}} & =\frac{d}{d t} \int_{\theta_{1}(t)}^{\theta_{2}(t)} L_{F} \beta_{0} e_{0} d z \\
& =\int_{\theta_{1}(t)}^{\theta_{2}(t)} \frac{d}{d t} L_{F} \beta_{0} e_{0} d z+\int_{\theta_{1}(t)}^{\theta_{2}(t)} L_{F} \frac{d}{d t} \beta_{0} e_{0} d z+L_{F}\left(\theta_{2}(t)\right) \beta_{0}(t) e_{0}\left(\theta_{2}(t)\right) \dot{\theta}_{2}(t) \\
& -L_{F}\left(\theta_{1}(t)\right) \beta_{0}(t) e_{0}\left(\theta_{1}(t)\right) \dot{\theta}_{1}(t) \\
& =\left\langle\frac{d}{d t} L_{F}, \beta_{0} e_{0}\right\rangle_{\underline{S}}+\left\langle L_{F}, \beta_{0, t} e_{0}\right\rangle_{\underline{S}}+L_{F}\left(\theta_{2}(t)\right) \beta_{0}(t) e_{0}\left(\theta_{2}(t)\right) \dot{\theta}_{2}(t) \\
& -L_{F}\left(\theta_{1}(t)\right) \beta_{0}(t) e_{0}\left(\theta_{1}(t)\right) \dot{\theta}_{1}(t) .
\end{aligned}
$$

We finally obtain that

$$
\begin{aligned}
\left\langle\frac{d}{d t} L_{F}, \beta_{0} e_{0}\right\rangle_{\underline{S}} & =\frac{d}{d t}\left\langle L_{F}, \beta_{0} e_{0}\right\rangle_{\underline{S}}-\frac{\beta_{0, t}}{\beta_{0}}\left\langle L_{F}, \beta_{0} e_{0}\right\rangle_{\underline{S}} \\
& -L_{F}\left(\theta_{2}(t)\right) \beta_{0}(t) e_{0}\left(\theta_{2}(t)\right) \dot{\theta}_{2}(t)+L_{F}\left(\theta_{1}(t)\right) \beta_{0}(t) e_{0}\left(\theta_{1}(t)\right) \dot{\theta}_{1}(t) .
\end{aligned}
$$

Replacing (19) and (23) into (22)

$$
\begin{aligned}
\frac{d}{d t}\left\langle L_{F}, \beta_{0} e_{0}\right\rangle_{\underline{S}} & =\left[\lambda_{0}-\left\langle(\phi N)^{\frac{\sigma-1}{\sigma}} N^{\frac{1}{\sigma}}\left(\beta_{0} e_{0}\right)^{-\frac{1}{\sigma}}, \beta_{0} e_{0}\right\rangle_{\underline{S}}+\frac{\beta_{0, t}}{\beta_{0}}\right]\left\langle L_{F}, \beta_{0} e_{0}\right\rangle_{\underline{S}} \\
& +L_{F}\left(\theta_{2}(t)\right) \beta_{0}(t) e_{0}\left(\theta_{2}(t)\right) \dot{\theta}_{2}(t)-L_{F}\left(\theta_{1}(t)\right) \beta_{0}(t) e_{0}\left(\theta_{1}(t)\right) \dot{\theta}_{1}(t) .
\end{aligned}
$$

Using (20), and removing the time variable for simplicity

$$
\frac{d}{d t}\left\langle L_{F}, \beta_{0} e_{0}\right\rangle_{\underline{S}}=\left[\frac{\lambda_{0}-\rho}{\sigma}+\frac{\beta_{0, t}}{\beta_{0}}\right]\left\langle L_{F}, \beta_{0} e_{0}\right\rangle_{\underline{S}}+\beta_{0}\left[L_{F}\left(\theta_{2}\right) e_{0}\left(\theta_{2}\right) \dot{\theta}_{2}-L_{F}\left(\theta_{1}\right) e_{0}\left(\theta_{1}\right) \dot{\theta}_{1}\right] .
$$

We can solve (25) in two stages 
1. First, we solve the homogeneous equation

$$
\begin{aligned}
\left\langle L_{F}, \beta_{0} e_{0}\right\rangle_{\underline{S}} & =\left\langle L_{F}, \beta_{0} e_{0}\right\rangle_{\underline{S}(\tau)} e^{\int_{\tau}^{t}\left(\frac{\lambda_{0}-\rho}{\sigma}+\frac{\beta_{0, t}}{\beta_{0}}\right) d s} \\
& =\left\langle L_{F}(\tau), \beta_{0}(\tau) e_{0}\right\rangle e^{\frac{\lambda_{0}-\rho}{\sigma}(t-\tau)} e^{\int_{\tau}^{t} \frac{\beta_{0, t}}{\beta_{0}} d s}=\left\langle L_{F}(\tau), \beta_{0}(\tau) e_{0}\right\rangle e^{\frac{\lambda_{0}-\rho}{\sigma}(t-\tau)} e^{\left.\ln \beta_{0}\right|_{\tau} ^{t}} \\
& =\left\langle L_{F}(\tau), \beta_{0}(\tau) e_{0}\right\rangle e^{\frac{\lambda_{0}-\rho}{\sigma}(t-\tau)} e^{\left.\ln \beta_{0}\right|_{\tau} ^{t}}=\left\langle L_{F}(\tau), \beta_{0}(\tau) e_{0}\right\rangle e^{\frac{\lambda_{0}-\rho}{\sigma}(t-\tau)} \frac{\beta_{0}(t)}{\beta_{0}(\tau)} \\
& =\left\langle L_{F}(\tau), \alpha_{0} e_{0}\right\rangle e^{\frac{\lambda_{0}-\rho}{\sigma}(t-\tau)} \frac{\beta_{0}(t)}{\alpha_{0}}
\end{aligned}
$$

where $L_{F}(\tau)$ is the distribution of fertile soil at the time the second phase is triggered and $\underline{S}(\tau)=\mathcal{S}$. By continuity, it is also true that $\beta_{0}(\tau)=\alpha_{0}$.

2. Then we solve the non-homogeneous equation trying a solution of the type

$$
\left\langle L_{F}, \beta_{0} e_{0}\right\rangle_{\underline{S}}=m(t) e^{\frac{\lambda_{0}-\rho}{\sigma} t} \beta_{0}(t)
$$

whose time derivative is

$$
\begin{aligned}
\frac{d}{d t}\left\langle L_{F}, \beta_{0} e_{0}\right\rangle_{\underline{S}} & =\dot{m}(t) e^{\frac{\lambda_{0}-\rho}{\sigma} t} \beta_{0}+m(t) \frac{\lambda_{0}-\rho}{\sigma} e^{\frac{\lambda_{0}-\rho}{\sigma} t} \beta_{0}+m(t) e^{\frac{\lambda_{0}-\rho}{\sigma} t} \beta_{0, t}(t) \\
& =e^{\frac{\lambda_{0}-\rho}{\sigma} t} \beta_{0}\left[\dot{m}(t)+m(t)\left(\frac{\lambda_{0}-\rho}{\sigma}+\frac{\beta_{0, t}(t)}{\beta_{0}(t)}\right)\right] .
\end{aligned}
$$

If (27) is a solution, then it has to verify (25). Replacing $\left\langle L_{F}, \beta_{0} e_{0}\right\rangle_{\underline{S}}$ by (27) and $\frac{d}{d t}\left\langle L_{F}, \beta_{0} e_{0}\right\rangle_{\underline{S}}$ using (28), we get that

$$
\dot{m}(t)=e^{-\frac{\lambda_{0}-\rho}{\sigma} t}\left[L_{F}\left(\theta_{2}\right) e_{0}\left(\theta_{2}\right) \dot{\theta}_{2}-L_{F}\left(\theta_{1}\right) e_{0}\left(\theta_{1}\right) \dot{\theta}_{1}\right]
$$

that is

$$
m(t)=\int_{\tau}^{t} e^{-\frac{\lambda_{0}-\rho}{\sigma} s}\left[L_{F}\left(\theta_{2}(s)\right) e_{0}\left(\theta_{2}(s)\right) \dot{\theta}_{2}(s)-L_{F}\left(\theta_{1}(s)\right) e_{0}\left(\theta_{1}(s)\right) \dot{\theta}_{1}(s)\right] d s .
$$

Plugging $m$ back into (27), and taking into account that $\left\langle L_{F}, \beta_{0} e_{0}\right\rangle_{\underline{S}(\tau)}=\left\langle L_{F}(\tau), \alpha_{0} e_{0}\right\rangle$, we obtain that the solution for $\left\langle L_{F}, \beta_{0} e_{0}\right\rangle_{\underline{S}}$ is

$$
\begin{aligned}
\left\langle L_{F}, \beta_{0} e_{0}\right\rangle_{\underline{S}} & =\frac{\beta_{0}(t)}{\alpha_{0}}\left\langle L_{F}(\tau), \alpha_{0} e_{0}\right\rangle e^{\frac{\lambda_{0}-\rho}{\sigma}(t-\tau)} \\
& +e^{\frac{\lambda_{0}-\rho}{\sigma} t} \beta_{0}(t) \int_{\tau}^{t} e^{-\frac{\lambda_{0}-\rho}{\sigma} s}\left[L_{F}\left(\theta_{2}(s)\right) e_{0}\left(\theta_{2}(s)\right) \dot{\theta}_{2}(s)-L_{F}\left(\theta_{1}(s)\right) e_{0}\left(\theta_{1}(s)\right) \dot{\theta}_{1}(s)\right] d s
\end{aligned}
$$

or

$$
\begin{aligned}
\left\langle L_{F}, \beta_{0} e_{0}\right\rangle_{\underline{S}} & =\beta_{0}(t)\left\langle L_{F}(\tau), e_{0}\right\rangle e^{\frac{\lambda_{0}-\rho}{\sigma}(t-\tau)} \\
& +\beta_{0}(t) \int_{\tau}^{t} e^{\frac{\lambda_{0}-\rho}{\sigma}(t-s)}\left[L_{F}\left(\theta_{2}(s)\right) e_{0}\left(\theta_{2}(s)\right) \dot{\theta}_{2}(s)-L_{F}\left(\theta_{1}(s)\right) e_{0}\left(\theta_{1}(s)\right) \dot{\theta}_{1}(s)\right] d s .
\end{aligned}
$$

With this solution for $\left\langle L_{F}, \beta_{0} e_{0}\right\rangle_{\underline{S}}$, we can obtain the optimal solution for consumption:

$$
c^{*}=\left[\phi \nabla v\left(L_{F}\right)\right]^{-\frac{1}{\sigma}}=\phi^{-\frac{1}{\sigma}}\left[\left\langle L_{F}, \beta_{0} e_{0}\right\rangle_{\underline{S}}^{-\sigma} \beta_{0} e_{0}\right]^{-\frac{1}{\sigma}}=\left(\phi \beta_{0} e_{0}\right)^{-\frac{1}{\sigma}}\left\langle L_{F}, \beta_{0} e_{0}\right\rangle_{\underline{S}} .
$$


Let us prove next that $c^{*}$ can be the optimal solution in Phase 2 under certain conditions. ${ }^{13}$ According to $I I$, optimal consumption in Phase 2 needs to be equal to $(\phi \psi)^{-1 / \sigma}$. On the other hand, the candidate we have just obtained is $c^{*}=\left(\phi \nabla v\left(L_{F}\right)\right)^{-1 / \sigma}$. Hence, to prove that $c^{*}$ is the optimal solution for consumption, it remains to prove that $\psi=\nabla v\left(L_{F}\right)$.

From the first order optimal conditions we know that $\psi(t, \theta)=\frac{1}{\phi(\theta)} c(t, \theta)^{-\sigma}$. We can substitute $c^{*}$ using (29) to obtain that

$$
\psi(t, \theta)=\beta_{0} e_{0}\left\langle L_{F}, \beta_{0} e_{0}\right\rangle_{\underline{S}}^{-\sigma}
$$

Using (30), the partial derivative of $\psi$ with respect to $t$ is

$$
\psi_{t}(t, \theta)=-\sigma \beta_{0} e_{0}\left\langle L_{F}, \beta_{0} e_{0}\right\rangle_{\underline{S}}^{-\sigma-1} \frac{d}{d t}\left\langle L_{F}, \beta_{0} e_{0}\right\rangle_{\underline{S}}+\beta_{0, t} e_{0}\left\langle L_{F}, \beta_{0} e_{0}\right\rangle_{\underline{S}}^{-\sigma}
$$

Note that we also know that $\mathcal{L} \psi(t, \theta)=\lambda_{0} \psi(t, \theta)$. Substituting $\psi_{t}$ and $\mathcal{L} \psi$ into the optimal condition for $\psi$

$$
\psi_{t}(t, \theta)+\mathcal{L} \psi(t, \theta)-\rho \psi(t, \theta)=0
$$

we have

$$
\begin{aligned}
& -\sigma \beta_{0} e_{0}\left\langle L_{F}, \beta_{0} e_{0}\right\rangle_{\underline{S}}^{-\sigma-1} \frac{d}{d t}\left\langle L_{F}, \beta_{0} e_{0}\right\rangle_{\underline{S}}+\beta_{0, t} e_{0}\left\langle L_{F}, \beta_{0} e_{0}\right\rangle_{\underline{S}}^{-\sigma}+\left(\lambda_{0}-\rho\right) \beta_{0} e_{0}\left\langle L_{F}, \beta_{0} e_{0}\right\rangle_{\underline{S}}^{-\sigma}=0 \\
& -\sigma \beta_{0} e_{0}\left\langle L_{F}, \beta_{0} e_{0}\right\rangle_{\underline{S}}^{-\sigma-1} \frac{d}{d t}\left\langle L_{F}, \beta_{0} e_{0}\right\rangle_{\underline{S}}+\left(\lambda_{0}-\rho+\frac{\beta_{0, t}}{\beta_{0}}\right) \beta_{0} e_{0}\left\langle L_{F}, \beta_{0} e_{0}\right\rangle_{\underline{S}}^{-\sigma}=0 \\
& -\sigma \frac{d}{d t}\left\langle L_{F}, \beta_{0} e_{0}\right\rangle_{\underline{S}}+\left(\lambda_{0}-\rho+\frac{\beta_{0, t}}{\beta_{0}}\right)\left\langle L_{F}, \beta_{0} e_{0}\right\rangle_{\underline{S}}=0 \\
& \frac{d}{d t}\left\langle L_{F}, \beta_{0} e_{0}\right\rangle_{\underline{S}}=\frac{1}{\sigma}\left(\lambda_{0}-\rho+\frac{\beta_{0, t}}{\beta_{0}}\right)\left\langle L_{F}, \beta_{0} e_{0}\right\rangle_{\underline{S}} .
\end{aligned}
$$

Hence, the solutions coming from the two approaches are identical if and only if

$$
\begin{gathered}
{\left[\frac{\lambda_{0}-\rho}{\sigma}+\frac{\beta_{0, t}}{\beta_{0}}\right]\left\langle L_{F}, \beta_{0} e_{0}\right\rangle_{\underline{S}}+\beta_{0}\left(L_{F}\left(\theta_{2}\right) e_{0}\left(\theta_{2}\right) \dot{\theta}_{2}-L_{F}\left(\theta_{1}\right) e_{0}\left(\theta_{1}\right) \dot{\theta}_{1}\right)} \\
=\frac{1}{\sigma}\left(\lambda_{0}-\rho+\frac{\beta_{0, t}}{\beta_{0}}\right)\left\langle L_{F}, \beta_{0} e_{0}\right\rangle_{\underline{S}} .
\end{gathered}
$$

The condition above can be simplified to

$$
\frac{1-\sigma}{\sigma} \frac{\beta_{0, t}}{\beta_{0}}\left\langle L_{F}, \beta_{0} e_{0}\right\rangle_{\underline{S}}=\beta_{0}\left(L_{F}\left(\theta_{2}\right) e_{0}\left(\theta_{2}\right) \dot{\theta}_{2}-L_{F}\left(\theta_{1}\right) e_{0}\left(\theta_{1}\right) \dot{\theta}_{1}\right) .
$$

In order to better understand our results, let us rewrite (21) as

$$
\frac{1-\sigma}{\sigma} \frac{\beta_{0, t}(t)}{\beta_{0}(t)}=\frac{N\left(\theta_{2}\right)\left[\phi\left(\theta_{2}\right) e_{0}\left(\theta_{2}\right)\right]^{-\frac{1-\sigma}{\sigma}} \dot{\theta}_{2}-N\left(\theta_{1}\right)\left[\phi\left(\theta_{1}\right) e_{0}\left(\theta_{1}\right)\right]^{-\frac{1-\sigma}{\sigma}} \dot{\theta}_{1}}{\left\langle\left(\phi e_{0}\right)^{-\frac{1-\sigma}{\sigma}} N, \mathbb{1}\right\rangle_{\underline{S}}}
$$

\footnotetext{
${ }^{13}$ Note that dynamic programming does not allow us to introduce the border conditions, $\dot{\theta}_{1}$ and $\dot{\theta}_{2}$. For this reason, we will mix here as in Appendix $\mathrm{C}$ dynamic programming and optimal control.
} 
Replacing $\frac{\beta_{0, t}}{\beta_{0}}$ into (31) using the expression above, we obtain that

$$
\begin{aligned}
& \dot{\theta_{2}}\left(\beta_{0} L_{F}\left(\theta_{2}\right) e_{0}\left(\theta_{2}\right)-\frac{\left\langle L_{F}, \beta_{0} e_{0}\right\rangle_{\underline{S}}}{\left\langle\left(\phi e_{0}\right)^{-\frac{1-\sigma}{\sigma}} N, \mathbb{1}\right\rangle_{\underline{S}}} N\left(\theta_{2}\right)\left(\phi\left(\theta_{2}\right) e_{0}\left(\theta_{2}\right)\right)^{\frac{\sigma-1}{\sigma}}\right) \\
& =\dot{\theta_{1}}\left(\beta_{0} L_{F}\left(\theta_{1}\right) e_{0}\left(\theta_{1}\right)-\frac{\left\langle L_{F}, \beta_{0} e_{0}\right\rangle_{\underline{S}}}{\left\langle\left(\phi e_{0}\right)^{-\frac{1-\sigma}{\sigma}} N, \underline{1}\right\rangle_{\underline{S}}} N\left(\theta_{1}\right)\left(\phi\left(\theta_{1}\right) e_{0}\left(\theta_{1}\right)\right)^{\frac{\sigma-1}{\sigma}}\right) .
\end{aligned}
$$

We can rewrite $(32)$ using the definition of $\theta_{i}$, i.e. $\dot{\theta}_{i}=\mathbf{D} L_{F, \theta}\left(\theta_{i}\right)$, and using that

$$
\psi(\theta)=\beta_{0} e_{0}(\theta)\left\langle L_{F}, \beta_{0} e_{0}\right\rangle_{\underline{S}}^{-\sigma}
$$

and

$$
\beta_{0}=\left(\frac{\sigma}{\rho-\lambda_{0}(1-\sigma)}\right)^{\frac{\sigma}{1-\sigma}}\left\langle\left(\phi e_{0}\right)^{-\frac{1-\sigma}{\sigma}} N, \mathbb{1}\right\rangle_{\underline{S}}^{\frac{\sigma}{1-\sigma}}
$$

to obtain

$$
\begin{aligned}
& L_{F, \theta}\left(\theta_{2}\right) e_{0}\left(\theta_{2}\right)\left(L\left(\theta_{2}\right)-\frac{\sigma}{\rho-\lambda_{0}(1-\sigma)} N\left(\theta_{2}\right) \phi\left(\theta_{2}\right)^{\frac{\sigma-1}{\sigma}} \psi\left(\theta_{2}\right)^{\frac{-1}{\sigma}}\right) \\
& =L_{F, \theta}\left(\theta_{1}\right) e_{0}\left(\theta_{1}\right)\left(L\left(\theta_{1}\right)-\frac{\sigma}{\rho-\lambda_{0}(1-\sigma)} N\left(\theta_{1}\right) \phi\left(\theta_{1}\right)^{\frac{\sigma-1}{\sigma}} \psi\left(\theta_{1}\right)^{\frac{-1}{\sigma}}\right) .
\end{aligned}
$$

(34) needs to be completed with the spatial boundary conditions that ensure that our operator is self-adjoint

$$
\left.\nabla_{\theta} L_{F}(z) \nabla v\left(L_{F}\right)\right|_{\theta_{1}(t)} ^{\theta_{2}(t)}=\left.L_{F}(z) \nabla_{\theta}\left(\nabla v\left(L_{F}\right)\right)\right|_{\theta_{1}(t)} ^{\theta_{2}(t)}
$$

that is:

$$
L_{F, \theta}\left(\theta_{2}\right) \psi\left(\theta_{2}\right)-L_{F, \theta}\left(\theta_{1}\right) \psi\left(\theta_{1}\right)=L\left(\theta_{2}\right) \psi_{\theta}\left(\theta_{2}\right)-L\left(\theta_{1}\right) \psi_{\theta}\left(\theta_{1}\right)
$$

Hence, the optimal solution for the co-state variable in Phase 2 is given by the following system made of a PDE for the shadow price of fertile land, two boundary conditions on $\psi$ and $\psi_{\theta}$ at $\theta_{1}$ and $\theta_{2}$, plus a transversality condition

$$
I I I\left\{\begin{array}{l}
\psi_{t}(t, \theta)+\mathcal{L} \psi(t, \theta)-\rho \psi(t, \theta)=0, \quad \forall t \geq \tau, \quad \text { and } \forall \theta \in \underline{S}, \\
L_{F, \theta}\left(\theta_{2}\right) e_{0}\left(\theta_{2}\right)\left(L\left(\theta_{2}\right)-\frac{\sigma}{\rho-\lambda_{0}(1-\sigma)} N\left(\theta_{2}\right) \lambda\left(\theta_{2}\right)^{\frac{\sigma-1}{\sigma}} \psi\left(\theta_{2}\right)^{\frac{-1}{\sigma}}\right) \\
=L_{F, \theta}\left(\theta_{1}\right) e_{0}\left(\theta_{1}\right)\left(L\left(\theta_{1}\right)-\frac{\sigma}{\rho-\lambda_{0}(1-\sigma)} N\left(\theta_{1}\right) \phi\left(\theta_{1}\right)^{\frac{\sigma-1}{\sigma}} \psi\left(\theta_{1}\right)^{\frac{-1}{\sigma}}\right), \quad \forall t \geq \tau, \\
L_{F, \theta}\left(\theta_{2}\right) \psi\left(\theta_{2}\right)-L_{F, \theta}\left(\theta_{1}\right) \psi\left(\theta_{1}\right)=L\left(\theta_{2}\right) \psi_{\theta}\left(\theta_{2}\right)-L\left(\theta_{1}\right) \psi_{\theta}\left(\theta_{1}\right), \quad \forall t \geq \tau, \\
\lim _{t \rightarrow \infty} \psi L_{F}=0, \quad \forall \theta \in \underline{S} .
\end{array}\right.
$$

\section{G. Proof of Proposition 5. Steady states in Phase 2.}

If a steady state is attained at time $\tau^{\prime} \geq 0$, then $\dot{\theta}_{1}(t)=\dot{\theta}_{2}(t)=0$ for all $t \geq \tau^{\prime}$. Let us denote the steady state solution $\left(\bar{L}_{F}, \bar{\psi}, \bar{\theta}_{1}, \bar{\theta}_{2}\right)$. At the steady state, the left hand side of (36) is zero. Since 
$L_{F}\left(\bar{\theta}_{i}\right)=L\left(\bar{\theta}_{i}\right)$ for $i=1,2,(36)$ implies that

$$
L\left(\bar{\theta}_{1}\right) e_{0, \theta}\left(\bar{\theta}_{1}\right)=L\left(\bar{\theta}_{2}\right) e_{0, \theta}\left(\bar{\theta}_{2}\right) .
$$

By definition of the steady state, $\bar{L}_{F}$ is the solution of

$$
\mathcal{L} \bar{L}_{F}=\phi N\left(\phi \beta_{0} e_{0}\right)^{-\frac{1}{\sigma}}\left\langle\bar{L}_{F}, \beta_{0} e_{0}\right\rangle_{\underline{S}},
$$

with $\bar{L}_{F}\left(\bar{\theta}_{1}\right)=L\left(\bar{\theta}_{1}\right)$ and $\bar{L}_{F}\left(\bar{\theta}_{2}\right)=L\left(\bar{\theta}_{2}\right)$. Besides, we know that $\bar{L}_{F, \theta}\left(\bar{\theta}_{2}\right)=\bar{L}_{F, \theta}\left(\bar{\theta}_{1}\right)=0$. $\bar{c}$ will obtain using that $\bar{\psi}=\frac{1}{\phi(\theta)} \bar{c}^{-\sigma}$. According to $I I I, \bar{\psi}$ is a solution to

$$
\mathcal{L} \bar{\psi}-\rho \bar{\psi}=0
$$

To complete the description of the steady state, we need the border conditions for $\bar{c}$ and $\bar{\psi} \cdot \bar{c}\left(\bar{\theta}_{1}\right)$ and $\bar{c}\left(\bar{\theta}_{2}\right)$ follow from (32) and (33). Note that (32) holds if the parenthesis are zero. Let us develop for $\bar{\theta}_{1}$, knowing that computations are identical for $\bar{\theta}_{2}$. Then, from (32)

$$
\beta_{0} L_{F}\left(\theta_{1}\right)=\frac{\left\langle L_{F}, \beta_{0} e_{0}\right\rangle_{\underline{S}}}{\left\langle\left(\phi e_{0}\right)^{-\frac{1-\sigma}{\sigma}} N, \mathbb{1}\right\rangle_{\underline{S}}} N\left(\theta_{1}\right)\left(\phi\left(\theta_{1}\right) e_{0}\left(\theta_{1}\right)\right)^{\frac{\sigma-1}{\sigma}} .
$$

Since $c\left(\theta_{1}\right)=\left\langle L_{F}, \beta_{0} e_{0}\right\rangle_{\underline{S}}\left(\phi\left(\theta_{1}\right) \beta_{0} e_{0}\left(\theta_{1}\right)\right)^{\frac{-1}{\sigma}}$, then

$$
\beta_{0} L_{F}\left(\theta_{1}\right)\left\langle\left(\phi e_{0}\right)^{-\frac{1-\sigma}{\sigma}} N, \mathbb{1}\right\rangle_{\underline{S}}=N\left(\theta_{1}\right) \phi\left(\theta_{1}\right) c \beta_{0}^{\frac{1}{\sigma}},
$$

so that

$$
c\left(\theta_{1}\right)=\frac{L_{F}\left(\theta_{1}\right)}{N\left(\theta_{1}\right) \phi} \beta_{0}^{\frac{\sigma-1}{\sigma}}\left\langle\left(\phi e_{0}\right)^{-\frac{1-\sigma}{\sigma}} N, \mathbb{1}\right\rangle_{\underline{S}} .
$$

Using (33), we obtain that

$$
\bar{c}\left(\bar{\theta}_{1}\right)=\frac{\rho-\lambda_{0}(1-\sigma)}{\sigma} \frac{L_{F}\left(\bar{\theta}_{1}\right)}{N\left(\bar{\theta}_{1}\right) \phi\left(\bar{\theta}_{1}\right)} \text { and } \bar{c}\left(\bar{\theta}_{2}\right)=\frac{\rho-\lambda_{0}(1-\sigma)}{\sigma} \frac{L_{F}\left(\bar{\theta}_{2}\right)}{N\left(\bar{\theta}_{2}\right) \phi\left(\bar{\theta}_{2}\right)} .
$$

The border conditions for $\bar{\psi}$ implied in (32) are

$$
\bar{\psi}\left(\bar{\theta}_{1}\right)=\frac{1}{\phi\left(\bar{\theta}_{1}\right)^{1-\sigma}}\left(\frac{\rho-\lambda_{0}(1-\sigma)}{\sigma} \frac{L_{F}\left(\bar{\theta}_{1}\right)}{N\left(\bar{\theta}_{1}\right)}\right)^{-\sigma} \text { and } \bar{\psi}\left(\bar{\theta}_{2}\right)=\frac{1}{\phi\left(\bar{\theta}_{2}\right)^{1-\sigma}}\left(\frac{\rho-\lambda_{0}(1-\sigma)}{\sigma} \frac{L_{F}\left(\bar{\theta}_{2}\right)}{N\left(\bar{\theta}_{2}\right)}\right)^{-\sigma} .
$$

Hence the steady state $\left(\bar{L}_{F}, \bar{\psi}, \bar{\theta}_{1}, \bar{\theta}_{2}\right)$ is the solution to 


$$
I V\left\{\begin{array}{l}
\mathcal{L} \bar{L}_{F}=\phi N\left(\phi \beta_{0} e_{0}\right)^{-\frac{1}{\sigma}}\left\langle\bar{L}_{F}, \beta_{0} e_{0}\right\rangle_{\underline{S}}, \\
\bar{L}_{F}\left(\bar{\theta}_{1}\right)=L\left(\bar{\theta}_{1}\right), \quad \bar{L}_{F}\left(\bar{\theta}_{2}\right)=L\left(\bar{\theta}_{2}\right), \\
L_{\theta}\left(\bar{\theta}_{1}\right)=0, \quad L_{\theta}\left(\bar{\theta}_{2}\right)=0, \\
\mathcal{L} \bar{\psi}-\rho \bar{\psi}=0, \\
\bar{\psi}\left(\bar{\theta}_{1}\right)=\frac{1}{\phi\left(\bar{\theta}_{1}\right)^{1-\sigma}}\left(\frac{\rho-\lambda_{0}(1-\sigma)}{\sigma} \frac{L_{F}\left(\bar{\theta}_{1}\right)}{N\left(\bar{\theta}_{1}\right)}\right)^{-\sigma}, \\
\bar{\psi}\left(\bar{\theta}_{2}\right)=\frac{1}{\phi\left(\bar{\theta}_{2}\right)^{1-\sigma}}\left(\frac{\rho-\lambda_{0}(1-\sigma)}{\sigma} \frac{L_{F}\left(\bar{\theta}_{2}\right)}{N\left(\bar{\theta}_{2}\right)}\right)^{-\sigma},
\end{array}\right.
$$

Recall that in Phase $2 \rho<\lambda_{0}=\max _{\lambda \in \sigma(\mathcal{L})} \lambda$. Since $\bar{\psi}$ solves $\mathcal{L} \psi-\rho \psi, \psi$ is an eigenvalue of $\mathcal{L}$. Additionally, we know that $\bar{\lambda} \neq 0$, meaning that there exists a non-zero steady state that we characterize next. Since the eigenvectors of $\mathcal{L}$ are orthogonal, then

$$
\begin{aligned}
\left\langle\bar{\psi}, e_{0}\right\rangle_{\underline{S}}=0 & \Longleftrightarrow\left\langle\frac{\bar{c}^{-\sigma}}{\phi}, e_{0}\right\rangle_{\underline{S}}=0 \Longleftrightarrow\left\langle\frac{\left[\left\langle\bar{L}_{F}, \beta_{0} e_{0}\right\rangle_{\underline{S}}\left(\phi \beta_{0} e_{0}\right)^{-\frac{1}{\sigma}}\right]^{-\sigma}}{\phi}, e_{0}\right\rangle_{\underline{S}}=0 \\
& \Longleftrightarrow\left\langle\bar{L}_{F}, \beta_{0} e_{0}\right\rangle_{\underline{S}}^{-\sigma} \beta_{0}\left\langle e_{0}, e_{0}\right\rangle_{\underline{S}}=0 \Longleftrightarrow\left\langle\bar{L}_{F}, \beta_{0} e_{0}\right\rangle_{\underline{S}}=0 .
\end{aligned}
$$

The last equality obtains because $\beta_{0} \neq 0$ and $\left\langle e_{0}, e_{0}\right\rangle_{S}=1$. Finally, since $\bar{L}_{F}$ is a solution to

$$
\mathcal{L} \bar{L}_{F}=\phi N\left(\phi \beta_{0} e_{0}\right)^{-\frac{1}{\sigma}}\left\langle\bar{L}_{F}, \beta_{0} e_{0}\right\rangle_{\underline{S}},
$$

and since $\left\langle\bar{L}_{F}, \beta_{0} e_{0}\right\rangle_{\underline{S}}=0$, we have that $\mathcal{L} \bar{L}_{F}=0$, which implies that $\bar{L}_{F} \in$ ker $\mathcal{L}$, i.e. $\bar{L}_{F}$ is a solution to $\mathcal{L} \bar{L}_{F}=0 \Longleftrightarrow D \bar{L}_{F}^{\prime \prime}+A(\phi-\nu) \bar{L}_{F}=0$. 